\title{
THE CHARACTER OF PLANAR TESSELLATIONS WHICH ARE NOT SIDE-TO-SIDE
}

\author{
RICHARD COWAN ${ }^{1}$ AND CHRISTOPH THÄLE ${ }^{\bigotimes, 2}$ \\ ${ }^{1}$ School of Mathematics and Statistics, University of Sydney, NSW 2006, Australia; ${ }^{2}$ Faculty of Mathematics, \\ Ruhr-University Bochum, Germany \\ e-mail: rcowan@usyd.edu.au, cthaele@uos.de \\ (Received July 23, 2013; revised December 3, 2013; accepted December 15, 2013)
}

\begin{abstract}
This paper studies stationary tessellations and tilings of the plane in which all cells are convex polygons. The focus is on the class of tessellations which are not side-to-side. The character of these tessellations is explored, with special attention paid to the relationship between edges of the tessellation and sides of the polygonal cells and to the combinatorial topology between the 'adjacent' geometric elements of the tessellation. Three new parameters, $\varepsilon_{0}, \varepsilon_{1}$ and $\varepsilon_{2}$ summing to unity, are introduced. These capture the essence of non side-to-side tessellations and play a role in understanding the adjacency of sides and cells. Examples illustrate the theory.
\end{abstract}

Keywords: combinatorial topology, Delaunay tessellation, random tessellations, STIT tessellation, stochastic geometry, tilings.

\section{INTRODUCTION}

As discussed in the recent paper of Weiss and Cowan (2011), the focus of attention in most studies of planar tessellations and tilings has been the side-to-side case, where each side of a polygonal cell coincides with a neighbouring cell's side. The studies of Cowan $(1978 ; 1979)$ are early exceptions in the random tessellation theory. Those studies, which also have relevance to the tiling literature, introduced a new parameter $\phi$. It quantified one of the most important features of non side-to-side tessellations, namely the occurrence of a type of vertex not seen in the simpler side-to-side case: the so-called $\pi$-vertex. If a vertex has $j$ emanating edges, there are $j$ angles subtended by these edges at the vertex. If one of these angles is equal to $\pi$, the vertex is called a $\pi$-vertex. A vertex that is not a $\pi$-vertex is called a $\bar{\pi}$-vertex. The parameter $\phi$ is defined as the proportion of vertices which are $\pi$-vertices.

Many properties of a non side-to-side tessellation can be expressed in terms of $\phi$ together with another parameter, $\theta$, the mean number of emanating edges from the typical vertex. These fundamental parameters, which capture both topological and combinatorial aspects, satisfy the general constraints

$$
0 \leq \phi \leq 1 ; \quad 3 \leq \theta \leq 6-2 \phi,
$$

as proved in Weiss and Cowan (2011) and illustrated in Fig. 5a. There are, however, some entities of a combinatorial and/or topological nature which cannot be expressed as a function of $\theta$ and $\phi$. So it is clear that other parameters of the non side-to-side tessellation are important. In this paper, we investigate these issues, delving into some of the finer structure of tessellations which are not side-to-side. Some metric issues are also discussed and one parameter, the mean length of the typical tessellation edge, plays a prominent role.

Fig. 1 illustrates that a general random tessellation allows any shape or size of convex polygon to be a cell; furthermore it potentially allows any number and geometry of edges emanating from a tessellation vertex. Some models, however, might restrict the variation of these features somewhat.

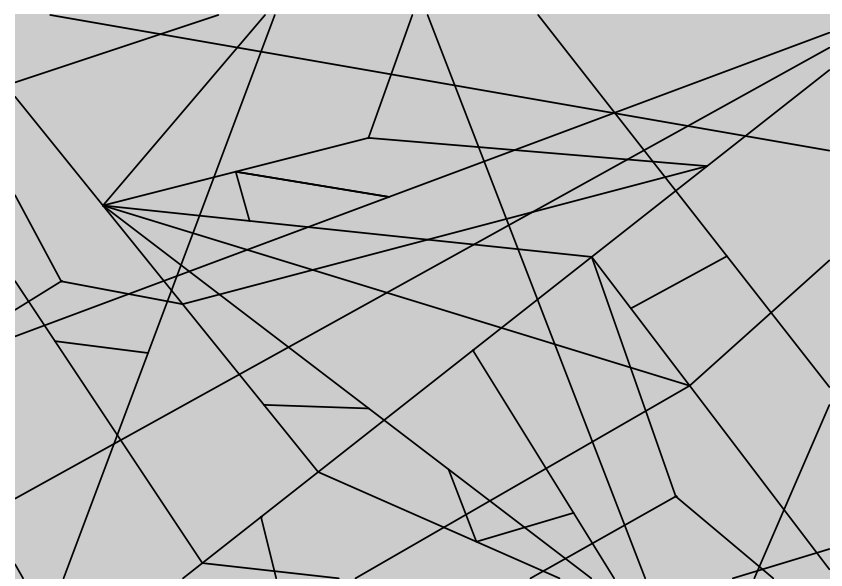

Fig. 1. Illustration of vertex complexity in a non side-to-side planar tessellation. A proportion are $\pi$ vertices. Also seen are many cell-sides which do not coincide with a side of a neighbouring cell. 


\section{GEOMETRIC OBJECTS AND THEIR ADJACENCY}

A tessellation of the plane is a collection of compact convex polygonal cells which cover the plane and overlap only on their boundaries. The union of the cell boundaries is called the tessellation frame. Each cell has sides and corners, these being respectively the 1 -faces and 0 -faces of the polygon, which lie on the frame. The union (taken over all cells) of the cell's corners is a collection of points in the plane called the vertices of the tessellation. Those line segments which are contained in the frame, have a vertex at each end and no vertices in their interior are called edges of the tessellation.

Two cells are said to be equal under motion if one can be found by translating and/or rotating the other. A tiling is a tessellation with a finite number of equivalence classes under this 'motion' relationship. Often a tiling seen in the literature has no randomness, but we also permit tilings generated randomly. Most tilings use only a small number of polygons with congruent copies of these assembled to cover the plane.

Our random planar tessellations (or planar tilings) are assumed to be stationary - and also locally-finite (avoiding points and line-segments 'accumulating').

The stationarity condition says that the geometric features are statistically invariant under translation. To achieve this with a non-random tiling, such as in Fig. 6, one must ensure that the planar origin is uniformly distributed in the repeating sub-unit. Hybrid models (partly random, partly non-random) can arise too, for example in the $2 \times 1$ tiling example in a later section; in that case, stationarity is created if the origin is uniformly distributed inside one of the original $2 \times 2$ squares.

The tessellation's primitive geometric elements, treated as compact domains, are the vertices, edges and cells. The sets of these elements are denoted by $V, E$ and $Z$ (for Zellen) respectively. These are the tessellation elements studied in the standard texts (Stoyan et al., 1995; Schneider and Weil, 2008). In this paper, however, other compact geometric elements will be introduced and the set of these will also be denoted by an upper-case letter - for example, the sets $S$ and $C$ of all sides and corners of cells, respectively.

We note that, for those elements which are not primitive but are instead derived from a primitive element, then the set may indeed be a multi-set. For example, in side-to-side tessellations, every $s \in S$ equals another member of $S$, as befits the terminology side-to-side. This may be true for some (but not all) members of $S$ in the non side-to-side case.

Generic sets of compact geometric objects are denoted by $X$ and $Y$. Subsets of the set $X$ are denoted by $X[\cdot]$, with the contents of the $[\cdot]$ being a suitably suggestive symbol introduced in an ad hoc manner. For example, we denote the sub-class of $\pi$-vertices by $V[\pi]$ and the subclass of $\bar{\pi}$-vertices by $V[\bar{\pi}]$.

Point processes and their intensities: The centroids of all members in a set of objects form a stationary point process on the plane, but this process might also have point multiplicity and, if so, it would not be a simple point process.

DEFINITION 1: The intensity of objects belonging to class $X$ is the intensity of the point process in $\mathrm{R}^{2}$ formed by centroids of the elements of X. It is denoted by $\lambda_{X}$.

The scale of the tessellation is determined by one of the intensity parameters and we have chosen $\lambda_{V}$ to play this scaling role. Our locally-finite condition implies that $\lambda_{V}$ is finite. The other main intensities, reported in Weiss and Cowan (2011), are expressed as follows in terms of $\lambda_{V}$ and are also finite:

$$
\lambda_{E}=\frac{\theta}{2} \lambda_{V}, \quad \lambda_{Z}=\frac{\theta-2}{2} \lambda_{V}, \quad \lambda_{S}=(\theta-\phi) \lambda_{V},
$$

with $\lambda_{C}=\lambda_{S}$. From these identities, we have

$$
\lambda_{S}=\frac{2(\theta-\phi)}{\theta} \lambda_{E}
$$

showing clearly the way this changes from $\lambda_{S}=2 \lambda_{E}$ as we move away from the side-to-side case which has $\phi=0$.

Metric parameters: There are many classes of line-segment that can be defined in a tessellation. The lengths of these segments together with the perimeters and areas of cells are the most obvious metric properties relevant to our study. The following notation applies.

DEFINITION 2: If the class $X$ comprises elements which are line-segments, we let $\bar{\ell}_{X}$ be the mean length of these segments. In particular, $\bar{\ell}_{E}$ and $\bar{\ell}_{S}$ are the mean edge length and mean side length respectively. We let $\bar{\ell}_{Z}$ be the mean cell perimeter and $\bar{a}_{Z}$ be the mean cell area.

It is known, from Cowan (1978), that $\bar{a}_{Z}=1 / \lambda_{Z}=$ $2 /\left(\lambda_{V}(\theta-2)\right)$ so $\bar{a}_{Z}$ is not a new parameter. The other metric entities $\bar{\ell}_{E}, \bar{\ell}_{S}$ and $\bar{\ell}_{Z}$ are related to the frame intensity. The frame $\mathscr{Y}$ of the tessellation is the union of all edges and its intensity is the mean total length of $\mathscr{Y}$ 's line-segments in any reference window $W$ of 
unit area. The text of Stoyan et al. (1995) shows that the frame intensity equals both $\lambda_{E} \bar{\ell}_{E}$ and $\frac{1}{2} \lambda_{Z} \bar{\ell}_{Z}$, so

$$
\bar{\ell}_{Z}=2 \frac{\lambda_{E}}{\lambda_{Z}} \bar{\ell}_{E}=\frac{2 \theta}{\theta-2} \bar{\ell}_{E},
$$

to which we add frame intensity $=\frac{1}{2} \lambda_{S} \bar{\ell}_{S}$ and the new formula

$$
\bar{\ell}_{S}=2 \frac{\lambda_{E}}{\lambda_{S}} \bar{\ell}_{E}=\frac{\theta}{\theta-\phi} \bar{\ell}_{E} .
$$

We see in Eq. 5 how the mean side length $\bar{\ell}_{S}$ departs from equality with the mean edge length $\bar{\ell}_{E}$ as $\phi$ becomes positive (that is, as the tessellation departs from the side-to-side case). These metric entities are inter-related; in this paper we have chosen $\bar{\ell}_{E}$ as the principal metric parameter.

Window formulae and transects: Other properties of the tessellation observed in a window $W$, chosen without reference to the tessellation itself, are known (see formula (3.4) in Cowan, 1978). The most interesting of these are for convex $W$ when the tessellation process $\mathscr{Y}$ is assumed isotropic (that is, its geometric features are statistically invariant under any planar rotation). In this case, the number of edges of the tessellation intersecting $W$ has expectation

$$
\mathbb{E} \mathrm{K}_{W}(E)=\left(\frac{\bar{\ell}_{E}}{\pi} \operatorname{Perim}(W)+\operatorname{Area}(W)\right) \lambda_{E} .
$$

Here we use the notation $\mathrm{K}_{W}(X)$, defined as the count of $X$-type objects intersecting $W$. Also the number of cells intersecting $W$ has expectation

$$
\begin{aligned}
& \mathbb{E} \mathrm{K}_{W}(Z)=1+ \\
&\left(\frac{\bar{\ell}_{E}}{\pi} \operatorname{Perim}(W)+\frac{\theta-2}{\theta} \operatorname{Area}(W)\right) \lambda_{E} .
\end{aligned}
$$

To these we now add the expected number of the tessellation's line-segments of a given class $X$ which intersect $W$ :

$$
\mathbb{E} \mathrm{K}_{W}(X)=\left(\frac{\bar{\ell}_{X}}{\pi} \operatorname{Perim}(W)+\operatorname{Area}(W)\right) \lambda_{X},
$$

as shown by formula (6) of Cowan (1979), a paper that deals with general isotropic line-segment processes.

When $W$ is a line-segment, of length $\ell$ say, then each of the window formulae simplify, because $\operatorname{Area}(W)=0$ and $\operatorname{Perim}(W)=2 \ell$.

REMARK 1: With such $a W$ and letting $\ell \rightarrow \infty$, Eq. 6 indicates informally that the point process formed by the intersection of tessellation edges with a line transect (chosen without reference to the tessellation) has intensity $2 \bar{\ell}_{E} \lambda_{E} / \pi$. In some tessellation models, the point process on this transect is a Poisson process and then the number $\mathrm{K}_{W}(E)$ of edges hitting a line-segment $W$ of length $\ell$ has the property

$$
\mathbb{P}\left\{\mathrm{K}_{W}(E)=k \mid \ell\right\}=\frac{(\rho \ell)^{k} \mathrm{e}^{-\rho \ell}}{k !},
$$

where

$$
\rho=\frac{2}{\pi} \bar{\ell}_{E} \lambda_{E} .
$$

In these 'Poisson Transect models', the Buffon-Laplace problem is effectively solved, giving a chance $e^{-\rho \ell}$ that a needle of length $\ell$ thrown randomly onto the tessellation 9 lies wholly within a cell of the tessellation. To understand this assertion, note that the thrown needle $W$ is assumed to be an isotropic random set. Eqs. 6-8, with expectations taken of the perimeter and area of $W$, are valid when $W$ is an isotropic random set even if $\mathscr{Y}$ is not isotropic.

Adjacency: The standard texts (Stoyan et al., 1995; Schneider and Weil, 2008) discuss only the primitive elements $V, E$ and $Z$. Their notation which works well in that restricted context is unsuitable when other object classes like $S$ or $C$ are introduced, because then object classes are not uniquely defined by their dimension. For example, the textbook notation $\lambda_{1}$ used for the intensity of edges $E$ (because edge have dimension one) cannot be used when there is another object class, sides $S$, whose elements also have dimension one. This notational deficiency becomes serious for tessellations in $\mathrm{R}^{3}$ (Weiss and Cowan, 2011) but, even in $R^{2}$, we find advantage in adopting the notation used in Weiss and Cowan (2011). We recommend to the reader the first two sections of their paper, as it provides further discussion and motivation for the notation.

Definition 3: An object $x \in X$ is said to be adjacent to an object $y \in Y$ if either $x \subseteq y$ or $y \subseteq x$. For any $x \in X$, the number of objects of type $Y$ adjacent to $x$ is denoted by $m_{Y}(x)$. For a random tessellation we define $\mu_{X Y}$ as the expected value of $m_{Y}(x)$ when $x$ is the typical member of $X$. Formally, we write $\mu_{X Y}:=\mathbb{E}_{X}\left(m_{Y}(x)\right)$, where the symbol $\mathbb{E}_{X}$ (and the probability measure $\mathbb{P}_{X}$ on which it is based) indicate that we are dealing with the typical element of type $X$ (Stoyan et al., 1995; Weiss and Cowan, 2011). The second moment $\mathbb{E}_{X}\left(m_{Y}(x)^{2}\right)$ of the number of type $Y$ objects adjacent to the typical $X$ object is written as $\mu_{X Y(2)}$.

Many features of interest can be expressed as an adjacency. For example, $\mu_{S E}$ is the expected number of edges adjacent to a typical side (that is, the mean number of edges in a typical side). This is one of 
the quantities expressible solely in terms of $\theta$ and $\phi$ (Weiss and Cowan, 2011):

$$
\mu_{S E}=\frac{\theta}{\theta-\phi},
$$

which clearly equals 1 in the side-to-side case.

The nine values of $\mu_{X Y}$ where both $X$ and $Y$ are primitive elements, that is $X$ and $Y \in\{V, E, Z\}$, can all be expressed solely in terms of $\theta$ (Stoyan et al., 1995; Weiss and Cowan, 2011). The most important two can be presented as follows.

$$
\mu_{Z E}=\mu_{Z V}=\frac{2 \theta}{\theta-2},
$$

which were first proven for the general case in Cowan $(1978 ; 1980)$ and Mecke $(1980)$, though they had been mentioned without proof and in restricted cases earlier. In the side-to-side case, the right-hand side of Eq. 10 also equals the expected number of sides (or corners) of a typical cell - but these entities take a different form in the non side-to-side case, as we discuss in a later subsection.

When $X$ and $Y$ are both primitive-element classes, it has been shown in Mecke (1980), Weiss and Zähle (1988) and Leistritz and Zähle (1992) that

$$
\lambda_{X} \mu_{X Y}=\lambda_{Y} \mu_{Y X},
$$

and this identity also holds when either $X$ or $Y$ or both are classes of faces of primitives; Møller's Theorem 5.1 (Møller, 1989) provides the proof of this extension. As an example,

$$
\mu_{S E}=\frac{\mu_{E S} \lambda_{E}}{\lambda_{S}}=\frac{\theta}{\theta-\phi},
$$

proving Eq. 9 with the use of Eq. 3 and the obvious fact $\mu_{E S}=2$.

In concluding this subsection, we note that $\theta$ and $\phi$ can be expressed using the adjacency notation; $\theta=\mu_{V E}$ and $\phi=\mu_{V S}^{\circ}$, the expected number of 'side-interiors' adjacent to a typical vertex (where the interior of a side, or indeed of any object $x$ of lower dimension than the space of the tessellation, is defined using the relative topology on $x$ ). Also $\stackrel{\circ}{X}$ denotes the class comprising the relative interiors of objects in class $X$. For brevity we drop the word 'relative' in the sequel; 'interior' means 'relative interior'.

Faces 'owned by' other objects: As proved in Cowan (1978; 1980) and used in applications (Cowan and Morris, 1988; Cowan and Tsang, 1994),

$$
v_{1}(Z)=v_{0}(Z)=\frac{2(\theta-\phi)}{\theta-2},
$$

where $v_{1}(Z)$ and $v_{0}(Z)$ are the mean number of sides and corners of the typical cell. This is an ownership concept rather than an adjacency; not all sides that are adjacent to a cell belong to it. The $v$ notation in Eq. 12 will be generically defined shortly in Definition 4, after we discuss an application.

Eq. 12 has application to the type of question common in the literature on tilings. "Can we tile the plane using only convex pentagonal tiles?". Obviously we can, there being examples using congruent copies of some particular pentagons (Grünbaum and Shephard, 1987). But Eq. 12, combined with Eq. 1, tells us that we certainly can't do so if $\phi>\frac{1}{2}$, even if we use convex pentagons of differing sizes and shapes (because then $\theta$ would be $<3$ ). The bounding case, with $\phi=\frac{1}{2}$ and $\theta=3$, can be realised so easily - start with the hexagonal lattice (made stationary in $R^{2}$ by placing the origin uniformly distributed within a hexagon) and divide every hexagon into two pentagons with a chord, ensuring that chord-ends never meet - that we might expect higher $\phi$ values are possible. But they are not!

DEFINITION 4: Let $X$ be a class of convex polytopes, all members of which have dimension $i$. For $j<i$, we define $n_{j}(x)$ as the number of $j$ faces of a particular object $x \in X$. Define $v_{j}(X):=$ $\mathbb{E}_{X}\left(n_{j}(x)\right)$, the expected number for the typical $X$ object. We define $X_{j}, j<i$, as the class of objects which are $j$-dimensional faces $(j$-faces) of some polytope belonging to $X$.

We shall use this notation in the planar case mainly when $j=0$ and $i=1$. For example, $E_{0}$ is the class of edge-termini and $S_{0}$ the class of side-termini. Also $Z_{0}$ and $Z_{1}$ are the classes of cell corners and sides respectively; also known by the labels $C$ and $S$ which we retain for convenience, except when defining the important entities, $v_{1}(Z)$ and $v_{0}(Z)$.

The disparity between the number of edges adjacent to a cell $z$ (that is, on the boundary of $z$ ) and the number of sides owned by $z$ provides another measure of departure from the side-to-side status. This suggested measure is $\mu_{Z E}-v_{1}(Z)$ which, from Eq. 10 and Eq. 12, equals $2 \phi /(\theta-2)$. This measure which we call the cell boundary disparity lies in the range $[0,2]$; the upper bound occurs in a number of models, notably in the STIT model which we discuss later in the paper.

\section{OTHER PARAMETERS AND THE EQUALITY RELATIONSHIP}

Two additional parameters: Sometimes the typical element of a class gives a biassed sampling of 
another type of element. Consider $\mu_{C E}$, the expected number of edges adjacent to a typical cell corner. The typical sampling of the corner gives a numberof-edges-emanating bias to the sampling of a vertex, and this leads to the introduction into the formula of $\mu_{V E(2)}$, the second moment of the 'number of edges emanating from the typical vertex'. As shown in Weiss and Cowan (2011),

$$
\mu_{C E}=\frac{\mu_{V E(2)}-\phi \mu_{V[\pi] E}}{\theta-\phi}, \quad \text { if } \phi>0,
$$

whilst $\mu_{C E}=\mu_{V E(2)} / \theta$ if $\phi=0$. Eq. 13 also introduces $\mu_{V[\pi] E}$, the expectation of the number edges emanating from the typical $\pi$-vertex, an entity defined when $\phi>$ 0 . For brevity, we denote it by $\theta_{\pi}$; we also use $\theta_{\bar{\pi}}$ for $\mu_{V[\bar{\pi}] E}$, though this is not another new parameter because

$$
\begin{aligned}
\theta \equiv \mu_{V E} & =\phi \mu_{V[\pi] E}+(1-\phi) \mu_{V[\bar{\pi}] E} \\
& \equiv \phi \theta_{\pi}+(1-\phi) \theta_{\bar{\pi}} .
\end{aligned}
$$

REMARK 2: Interestingly, whilst we see in Eq. 1 that $\theta$ (which $\equiv \mu_{V E}$ ) is bounded above, examples show that $\theta_{\pi}$ and $\theta_{\bar{\pi}}$ can be arbitrarily large. For example, it is easy to construct a tessellation with very few $\pi$ vertices with each $\pi$-vertex having $n$ edges, $n$ being arbitrarily large. Another example is available, with non- $\pi$ vertices being rare but emitting $n$ edges, where $n$ is large (details omitted).

Weiss and Cowan (2011) have used the four parameters $(\theta$ and $\phi$, together with the additional parameters, $\mu_{V E(2)}$ and $\theta_{\pi}$ ) to give formulae for all relevant intensity parameters $\lambda_{X}$ and for all but three of the forty-nine $\mu_{X Y}$ entities between the seven classes of elements that they study: $X$ and $Y$ both $\in\left\{E, V, Z, E_{0}, C, S, S_{0}\right\}$. This suggests that three more parameters are required, and we could choose to use the three missing entries in Weiss and Cowan (2011), $\mu_{S Z}, \mu_{Z S}$ and $\mu_{S S}-$ but actually two suffice because $\mu_{S Z}$ and $\mu_{Z S}$ are related (via Eq. 11). There are however more natural choices, as we shall see later in this section.

The equality relationship: The relationship between edge and side, and the cell 'owning' the side, will occupy much of our attention in this paper. The complications in their adjacency relationship is demonstrated in Fig. 2, perhaps explaining why formulae for the missing trio have not yet been found for the general non side-to-side tessellation, nor for any model until recently.

In the side-to-side case, $m_{S}(e)=2$ for every $e \in E$. Despite the added complication seen in Fig. 2 which arises in the non side-to-side case, this identity still holds - because adjacency involves the subset concept; every $e \in E$ is a subset $(\subseteq)$ of two sides. To better capture how edges and sides relate, we need to focus on another relationship, equality.

Definition 5: Suppose the objects in class $X$ have the same dimension as those in class $Y$. Then the number of objects of type $Y$ equal (as a set in $\mathrm{R}^{2}$ ) to some $x \in X$ is denoted by the random variable $\overline{\bar{m}}_{Y}(x)$. We define $\overline{\bar{\mu}}_{X Y}$ as $\mathbb{E}_{X}\left(\overline{\bar{m}}_{Y}(x)\right)$.

It is the loss of equality of $E$ and $S$, rather than their loss of adjacency, that happens when a tessellation is not side-to-side. An edge is not always equal to the side of two cells, that is, $\overline{\bar{m}}_{S}(e), e \in E$ may not always equal two; it takes the values 0,1 or 2 randomly. Depending on this value, the class $E$ can be divided into sub-classes $E[0], E[1]$ and $E[2]$.

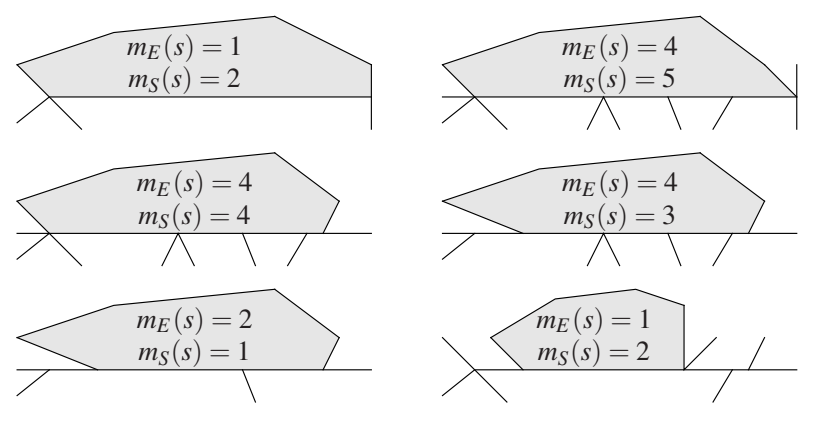

Fig. 2. The shaded region is a cell; one of its sides is the horizontal line-segment which bounds it below. This side s has a varying number $m_{E}(s)$ of edges contained within it, as shown. The value of $m_{S}(s)$, the number of sides adjacent to it (the count including sitself), also varies as shown.

We introduce, for the typical edge, the probabilities $\varepsilon_{0}, \varepsilon_{1}$ and $\varepsilon_{2}$ for these three outcomes. Naturally, $\varepsilon_{0}+$ $\varepsilon_{1}+\varepsilon_{2}=1$, and

$$
\overline{\bar{\mu}}_{E S}=\varepsilon_{1}+2 \varepsilon_{2} .
$$

When needed, we use symbols $\bar{\ell}_{E[0]}, \bar{\ell}_{E[1]}$ and $\bar{\ell}_{E[2]}$ for the expected lengths of typical edges of the three subclasses; obviously, $\varepsilon_{0} \bar{\ell}_{E[0]}+\varepsilon_{1} \bar{\ell}_{E[1]}+\varepsilon_{2} \bar{\ell}_{E[2]}=\bar{\ell}_{E}$.

We now consider the random variable $\overline{\bar{m}}_{E}(S)$, the number of edges equal to a typical side. It is binary, taking only the values in $\{0,1\}$. So, using Eq. 3 and Eq. 15,

$$
\begin{aligned}
\mathbb{P}_{S}\left\{\overline{\bar{m}}_{E}(s)=1\right\} & =\overline{\bar{\mu}}_{S E} \\
& =\frac{\lambda_{E}}{\lambda_{S}} \overline{\bar{\mu}}_{E S}=\frac{\theta\left(\varepsilon_{1}+2 \varepsilon_{2}\right)}{2(\theta-\phi)} .
\end{aligned}
$$


Also used in this expression is the analogue of Eq. 11; such analogues exist for all symmetric relations.

Another descriptor of 'not being side-to-side' is $\overline{\bar{\mu}}_{S S}$, the expected number of sides equal to a typical side. This is derived as follows:

$$
\overline{\bar{\mu}}_{S S}=1+\mu_{S E[2]}=1+\frac{2 \lambda_{E[2]}}{\lambda_{S}}=1+\frac{\varepsilon_{2} \theta}{\theta-\phi},
$$

using $\mu_{E[2] S}=2$ and Eq. 11 .

The missing mean adjacencies in terms of the epsilons: We now show that the three missing mean adjacencies, $\mu_{S Z}, \mu_{Z S}$ and $\mu_{S S}$, can be expressed in terms of $\theta, \phi, \varepsilon_{0}, \varepsilon_{1}$ and $\varepsilon_{2}$.

THEOREM 1: The three mean adjacencies missing in the table of forty-nine such entities in Weiss and Cowan (2011) are

$$
\begin{aligned}
& \mu_{S Z}=1+\frac{\theta\left(\varepsilon_{1}+2 \varepsilon_{2}\right)}{2(\theta-\phi)}, \\
& \mu_{Z S}=\frac{2(\theta-\phi)+\theta\left(\varepsilon_{1}+2 \varepsilon_{2}\right)}{\theta-2}, \quad \text { and } \\
& \mu_{S S}=1+\frac{\theta}{\theta-\phi}\left(1-\varepsilon_{0}\right) .
\end{aligned}
$$

Proof: Note that $m_{Z}(s)=1+\overline{\bar{m}}_{E}(s)$, for all $s \in S$, so $\mu_{S Z}=1+\overline{\bar{\mu}}_{S E}$ and Eq. 18 follows by applying Eq. 16. Furthermore, via Eq. 11, $\mu_{Z S}=\lambda_{S} \mu_{S Z} / \lambda_{Z}$ and this equals Eq. 19 using $\lambda_{Z}=\frac{1}{2}(\theta-2) \lambda_{V}$ and $\lambda_{S}=(\theta-\phi) \lambda_{V}$.

To prove Eq. 20, we start with a subtle expression for $m_{S}(s)$, the number of sides adjacent to a particular side $s$ (see Definition 3). For all sides $s$,

$$
m_{S}(s)=1+m_{E}(s)-m_{E[0]}(s) .
$$

The proof of this is essentially given by the diagrams in Fig. 2, treating the horizontal line-segment that bounds the shaded cell below as our particular $s$. Essentially, the six diagrams present all of the complexity that a neighbourhood of $s$ might have. It readily follows from Eq. 21 that

$$
\begin{aligned}
\mu_{S S} & =1+\mu_{S E}-\mu_{S E[0]} \\
& =1+\frac{\lambda_{E}}{\lambda_{S}} \mu_{E S}-\frac{\lambda_{E[0]}}{\lambda_{S}} \mu_{E[0] S \quad \text { using Eq. 11 }} \\
& =1+\frac{\lambda_{E}}{\lambda_{S}} \mu_{E S}-\frac{\lambda_{E[0]}}{\lambda_{E}} \frac{\lambda_{E}}{\lambda_{S}} \mu_{E[0] S} \\
& =1+\frac{2 \lambda_{E}}{\lambda_{S}}\left(1-\varepsilon_{0}\right) \\
& =1+\frac{\theta}{\theta-\phi}\left(1-\varepsilon_{0}\right),
\end{aligned}
$$

using the definition of $\varepsilon_{0}$, the fact that $\mu_{E S}=\mu_{E[0] S}=2$ and Eq. 3. Therefore, identity Eq. 20 has been proved and the theorem's proof is complete.

We consider that $\varepsilon_{0}, \varepsilon_{1}$ and $\varepsilon_{2}$ capture the essence of 'not being side-to-side', and so we adopt them as fundamental parameters instead of $\mu_{S Z}, \mu_{Z S}$ and $\mu_{S S}$, which are not as immediately relevant to the concept of side-to-side. Thus the topological parameter set of our choice now becomes $\left\{\theta, \phi, \mu_{V E(2)}, \theta_{\pi}, \varepsilon_{0}, \varepsilon_{1}, \varepsilon_{2}\right\}$, with $\varepsilon_{0}+\varepsilon_{1}+\varepsilon_{2}=1$. Our scale parameter $\lambda_{V}$ has relevance too, but it doesn't influence the combinatorial topology of our system. Nor does our main metric parameter $\bar{\ell}_{E}$ have topological relevance.

\section{EXAMPLES}

A $2 \times 1$ tiling: As a relatively simple learning example, consider the square lattice made up of $2 \times 2$ squares. Each square is then tiled by two $2 \times 1$ tiles, with random orientation for the long axis of these two tiles (vertical or horizontal with equal probability). The bold lines in Fig. 7 illustrate the construction. Find all the parameters of the tessellation - to reinforce the notation and the relationships between the parameters!

Looking at the typical cell, clearly $\bar{a}_{Z}=2, \bar{\ell}_{Z}=6$ and $v_{1}(Z)=4$. Therefore $\lambda_{Z}=1 / \bar{a}_{Z}=\frac{1}{2}$ and, from Eq. $12, \phi=4-\theta$. Note also that $\mu_{Z V}=\frac{9}{2}$, because on one side (and only one) of each tile there is an extra vertex added with probability $\frac{1}{2}$. Therefore, from Eq. $10, \theta=\frac{18}{5}$. Then $\phi=4-\theta=\frac{2}{5}$. Now we can write $\lambda_{V}=2 \lambda_{Z} /(\theta-2)=\frac{5}{8}$ and $\lambda_{E}=\theta \lambda_{V} / 2=\frac{9}{8}$, using Eq. 2. Also write $\lambda_{V[\pi]}=\phi \lambda_{V}=\frac{1}{4}$. It is obvious that $\varepsilon_{0}=0$ and that $\lambda_{E[1]}=2 \lambda_{V[\pi]}=\frac{1}{2}$. Therefore $\varepsilon_{1}=\lambda_{E[1]} / \lambda_{E}=\frac{4}{9}, \varepsilon_{2}=1-\varepsilon_{0}-\varepsilon_{1}=\frac{5}{9}$ and $\lambda_{E[2]}=$ $\varepsilon_{2} \lambda_{E}=\frac{5}{8}$. The line intensity is $\frac{1}{2} \bar{\ell}_{Z} \lambda_{Z}$, which equals $\frac{3}{2}$; the line intensity is also $\bar{\ell}_{E} \lambda_{E}$, so $\bar{\ell}_{E}=\frac{4}{3}$. As obviously $\bar{\ell}_{E[1]}=1$, we derive $\bar{\ell}_{E[2]}=\left(\bar{\ell}_{E}-\bar{\ell}_{E[1]} \lambda_{E[1]}\right) / \lambda_{E[2]}=\frac{4}{3}$. Finally $\theta_{\pi}=3$ and $\mu_{V E(2)}=9 \phi+16(1-\phi)=\frac{66}{5}$.

The STIT model: This tessellation, perhaps the best-known model that is not side-to-side, was first studied by Nagel and Weiss (2003; 2005), and later by them in collaboration with Mecke (Mecke et al., 2007; 2011). Other recent studies of the planar STIT tessellation are by Schreiber and Thäle $(2010 ; 2013)$ and Cowan (2013). In this model, drawn in Fig. 3a, all vertices are $\pi$-vertices with three edges emanating, so $\theta \equiv \mu_{V E}=\theta_{\pi}=3, \mu_{V E(2)}=9$ and $\phi=1$. Fortysix adjacency entities can now be evaluated from Weiss and Cowan (2011), the most interesting in the context of the current paper being $\mu_{S E}=\theta /(\theta-$ $\phi)=\frac{3}{2}$ and $\mu_{Z E}=2 \theta /(\theta-2)=6$. There is also the 
important $v$ entity, the mean number of sides for the typical cell:

$$
v_{1}(Z)=\frac{2(\theta-\phi)}{\theta-2}=4 .
$$

So the cell boundary disparity is 2 .

The results given above for the STIT model are not new (see Nagel and Weiss, 2005), but, until the recent paper (Cowan, 2013), STIT results for $\varepsilon_{0}, \varepsilon_{1}, \varepsilon_{2}, \bar{\ell}_{E[0]}, \bar{\ell}_{E[1]}, \bar{\ell}_{E[2]}, \mu_{S Z}, \mu_{Z S}$ and $\mu_{S S}$ were unknown. From this recent paper, we state the following values:

$$
\begin{aligned}
& \varepsilon_{0}=\frac{2}{3}(2 \log 2-1) \approx 0.25753, \\
& \varepsilon_{1}=\frac{2}{3}(5-6 \log 2) \approx 0.56075, \\
& \varepsilon_{2}=\frac{1}{3}(8 \log 2-5) \quad \approx 0.18173, \\
& \bar{\ell}_{E[0]}=\frac{3(3-4 \log 2)}{2(2 \log 2-1)} \bar{\ell}_{E} \approx 0.883 \bar{\ell}_{E}, \\
& \bar{\ell}_{E[1]}=\frac{3(8 \log 2-5)}{2(5-6 \log 2)} \bar{\ell}_{E} \approx 0.972 \bar{\ell}_{E}, \\
& \bar{\ell}_{E[2]}=\frac{3(3-4 \log 2)}{8 \log 2-5} \bar{\ell}_{E} \approx 1.251 \bar{\ell}_{E} .
\end{aligned}
$$

Here, $\bar{\ell}_{E}=\frac{1}{3} \sqrt{2 \pi / \lambda_{V}}$. From these values, together with Eqs. 15-19 from the current paper, we have the following results:

$$
\begin{aligned}
& \overline{\bar{\mu}}_{E S}=\varepsilon_{1}+2 \varepsilon_{2}=\frac{4}{3} \log 2 \approx 0.9242, \\
& \overline{\bar{\mu}}_{S E}=\mathbb{P}_{S}\left\{\overline{\bar{m}}_{E}(s)=1\right\}=\log 2 \approx 0.6931, \\
& \overline{\bar{\mu}}_{S S}=1+\frac{\varepsilon_{2} \theta}{\theta-\phi}=4 \log 2-\frac{3}{2} \approx 1.2726, \\
& \mu_{S Z}=1+\log 2 \approx 1.6931, \\
& \mu_{Z S}=4+\frac{4}{3} \log 2 \approx 4.9242 .
\end{aligned}
$$

Also derived in Cowan (2013) is

$$
\mu_{S S}=\frac{7}{2}-2 \log 2 \approx 2.1137,
$$

together with full distributions for many of the STIT adjacency relationships. It is readily seen that $\mu_{S S}$ calculated by the new formula, Eq. 20 in Theorem 1, agrees with the result in Cowan (2013).

Divided Delaunay: Start with a stationary and isotropic Delaunay tessellation $\mathscr{D}_{0}$ based on a planar Poisson point process with intensity $\rho$ (the dots in Fig. 3b). This tessellation is illustrated by the solid line segments in the figure, connecting 'neighbouring' Poisson dots (see the formal definition in Schneider and Weil (2008)). This tessellation is side-to-side, so has $\phi=0$. Also, since all cells are triangles, $v_{1}(Z)=3$ and therefore $\theta=6$ from Eq. 12. The second moment, $\mu_{V E(2)}$, introduced above, is known in the form of a complicated multiple integral which evaluates to be 37.7808 approximately (Heinrich and Muche, 2008). Obviously $\lambda_{V}=\rho$; then using Eq. 2 we get $\lambda_{E}=\frac{1}{2} \theta \lambda_{V}=3 \rho$ and $\lambda_{Z}=\frac{1}{2}(\theta-$ 2) $\lambda_{V}=2 \rho$. Also, because $\lambda_{E[2]}=\lambda_{E}$, it is clear that $\varepsilon_{2}=1$. Note that $\bar{\ell}_{E}=32 /(9 \pi \sqrt{\rho})$ (Miles, 1970).

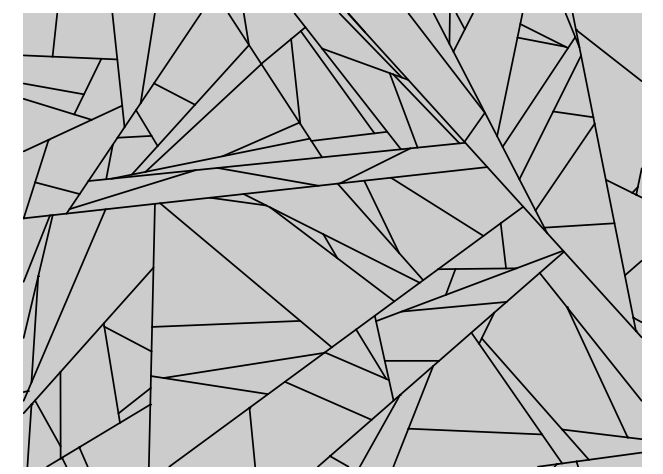

(a)

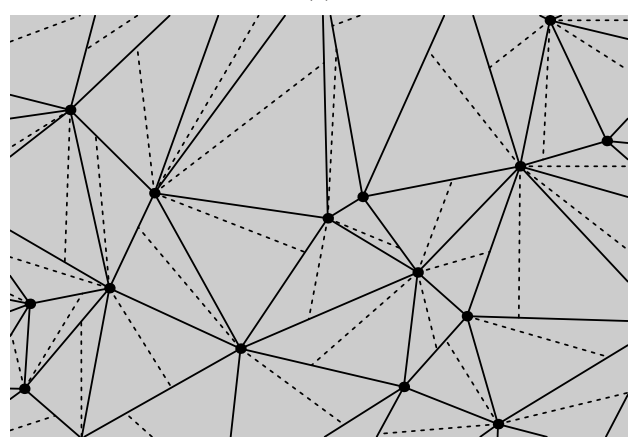

(b)

Fig. 3. (a) A realisation of the STIT model within a rectangular viewing window. (b) The 'divided Delaunay' model. The chords are shown as dashed lines.

In each of $\mathscr{D}_{0}$ 's triangular cells we independently choose a vertex (each equiprobable) and construct a chord from that vertex to a uniformly distributed point on the opposite side of the triangle (see the dotted line segments in Fig. 3b). We label the new tessellation $\mathscr{D}_{1}$. Further iteration using the same random division rules yields $\mathscr{D}_{2}, \mathscr{D}_{3}, \ldots$, each being a tessellation with only triangular cells. Using superscripts $(n)$ for the parameters of $\mathscr{D}_{n}$ (even when $n=0$ ), we note that if $n \geq 1$,

$$
\begin{array}{ll}
\lambda_{Z}^{(n)}=2 \lambda_{Z}^{(n-1)}, & \lambda_{V[\pi]}^{(n)}=\lambda_{Z}^{(n-1)}+\lambda_{V[\pi]}^{(n-1)}, \\
\lambda_{E}^{(n)}=2 \lambda_{Z}^{(n-1)}+\lambda_{E}^{(n-1)}, & \lambda_{V}^{(n)}=\lambda_{Z}^{(n-1)}+\lambda_{V}^{(n-1)}, \\
\theta^{(n)}=6-2 \phi^{(n)}, &
\end{array}
$$

the last of these identities arising from Eq. 12 (and holding for any tessellation which has only triangular 
cells). These four difference equations are readily solved to give

$$
\begin{array}{ll}
\lambda_{Z}^{(n)}=2^{n+1} \rho, & \lambda_{E}^{(n)}=\left(2^{n+2}-1\right) \rho, \\
\lambda_{V}^{(n)}=\left(2^{n+1}-1\right) \rho, & \lambda_{V[\pi]}^{(n)}=2\left(2^{n}-1\right) \rho,
\end{array}
$$

and these solutions yield a formula for $\phi^{(n)}$ which leads to $\theta^{(n)}$ :

$$
\begin{aligned}
\phi^{(n)}=\frac{\lambda_{V[\pi]}^{(n)}}{\lambda_{V}^{(n)}} & =\frac{2\left(2^{n}-1\right)}{2^{n+1}-1} \\
& \Longrightarrow \theta^{(n)}=6-2 \phi^{(n)}=2 \frac{2^{n+2}-1}{2^{n+1}-1} .
\end{aligned}
$$

Thus the cell boundary disparity is $1-2^{-n}$, which rises from zero to a limit of 1 as $n$ increases.

So for $\mathscr{D}_{1}, \lambda_{V}^{(1)}=3 \rho, \lambda_{E}^{(1)}=7 \rho, \phi^{(1)}=\frac{2}{3}$ and $\theta^{(1)}=\frac{14}{3}$. Also for $\mathscr{D}_{1}$ the calculation of the epsilons is easy because $\mathscr{D}_{0}$ is side-to-side and therefore has only $E[2]$ edges. An edge from $\mathscr{D}_{0}$ is divided into three segments with probability $1 / 9$, into two segments with probability $4 / 9$ and remains unchanged with probability $4 / 9$. Thus $\lambda_{E[2]}^{(1)}=\lambda_{Z}^{(0)}+1 \cdot \frac{4}{9} \lambda_{E[2]}^{(0)}=\frac{10}{3} \rho$, after allowing for the unchanged edges which remain as type $E[2]$. Furthermore, $\lambda_{E[0]}^{(1)}=\frac{1}{9} \lambda_{E}^{(0)}=\frac{1}{3} \rho$, as one type- $E[0]$ edge arises when an original edge is hit by two chords. Lastly, $\lambda_{E[1]}^{(1)}=\left(2 \cdot \frac{4}{9}+2 \cdot \frac{1}{9}\right) \lambda_{E}^{(0)}=\frac{10}{3} \rho$. Therefore, using $\varepsilon_{j}^{(1)}=\lambda_{E[j]}^{(1)} / \lambda_{E}^{(1)}$,

$$
\varepsilon_{0}^{(1)}=\frac{1}{21}, \quad \varepsilon_{1}^{(1)}=\frac{10}{21}, \quad \varepsilon_{2}^{(1)}=\frac{10}{21} .
$$

Then, using Theorem 1,

$$
\mu_{S Z}^{(1)}=\frac{11}{6}, \quad \mu_{Z S}^{(1)}=\frac{99}{28}, \quad \mu_{S S}^{(1)}=\frac{19}{9} .
$$

Additionally, from Eqs. 15-17,

$$
\overline{\bar{\mu}}_{E S}^{(1)}=\frac{10}{7}, \quad \overline{\bar{\mu}}_{S E}^{(1)}=\frac{5}{6}, \quad \overline{\bar{\mu}}_{S S}^{(1)}=\frac{14}{9} .
$$

For $\mathscr{D}_{1}$, one can also show that $\theta_{\pi}^{(1)}=3$ and $\mu_{V E(2)}^{(1)}=$ $\frac{58}{9}+\frac{16}{27} \times 37.7808 \approx 28.833$. Calculation of various entities for $\mathscr{D}_{n}$ when $n>1$, is more difficult and will be demonstrated later in the next section.

We close this example for now, noting that some limiting topological properties of repeatedly divided triangular cells have been addressed in Cowan (2004; 2010) whilst the three-dimensional version of dividing cells in a Delaunay tetrahedral tessellation is analysed in Weiss and Cowan (2011).

\section{FURTHER CLASSIFICATIONS OF EDGES AND EDGE-TERMINI}

We have seen that a typical edge may be equal to a variable number of sides, 0,1 or 2 . Is this variability also evident when we observe a typical edge-terminus?

A classification of edge-termini: We can sample a typical edge-terminus, that is a typical $e_{0} \in E_{0}$, by first sampling a typical edge $e \in E$ and then randomly choosing one of its termini. Having done this, we can observe the number of side-termini equal to the chosen edge-terminus - the count restricted to termini of sides $s$ such that $e \subseteq s$.

We see that such an $e_{0}$ is equal to either 1 or 2 side-termini. So each terminus can be sub-typed as $E_{0}[1]$ or $E_{0}[2]$ based on this idea. Introduce the temporary notation $\alpha$ for the expected number of such sides having the typical edge-terminus as an 0 face. Note that $\lambda_{E_{0}} \alpha=2 \lambda_{S}$, so $\alpha=2(\theta-\phi) / \theta$. This evaluates to $\frac{4}{3}$ in the STIT model and $\frac{12}{7}$ in the Divided Delaunay $\mathscr{D}_{1}$ model. Also, it is easily shown that $\lambda_{E_{0}[1]}=2 \lambda_{V[\pi]}=2 \phi \lambda_{V}$ (as there are two $E_{0}[1]$ termini at every $\pi$-vertex and none at vertices that are not $\pi$ vertices) and that $\lambda_{E_{0}[2]}=\lambda_{E_{0}}-\lambda_{E_{0}[1]}=(\theta-2 \phi) \lambda_{V}$. Thus

$$
\begin{aligned}
& \mathbb{P}_{E_{0}}\left\{e_{0} \in E_{0}[1]\right\}=\frac{2 \phi}{\theta}, \\
& \mathbb{P}_{E_{0}}\left\{e_{0} \in E_{0}[2]\right\}=1-\frac{2 \phi}{\theta} .
\end{aligned}
$$

Another classification of edges: An edge can be classified by the type of its termini. One potentially useful method might be as follows: an edge is of type $[j], j \in\{z e r o, o n e, t w o\}$ if exactly $j$ of its termini are of type $E_{0}[2]$. So this gives a new breakdown of $E$ into $E[z e r o], E[$ one $]$ and $E[t w o]$, a verbal annotation which avoids confusion with the previous numeric breakdown, $E[0], E[1]$ and $E[2]$. We note that $E[2] \equiv$ $E[t w o]$, so

$$
\mathbb{P}_{E}\{e \in E[t w o]\}=\varepsilon_{2} .
$$

We also note that $E[0] \subset E[$ zero $]$ and $E[$ one $] \subset E[1]$. Also each $E[1]$ edge can be labelled, 'zero' or 'one' from the second classification method. (see Fig. 4).

This means that the edges are now classified into four types - the original $E[2]$ and $E[0]$ plus the two components of $E[1]$, namely $E[1$ \& zero $]$ and $E[1 \&$ one $]$ which we abbreviate as $E[10]$ and $E[11]$ respectively. We also break $\varepsilon_{1}$ into two terms, $\varepsilon_{10}$ and $\varepsilon_{11}$, with $\varepsilon_{1}=\varepsilon_{10}+\varepsilon_{11}$. 


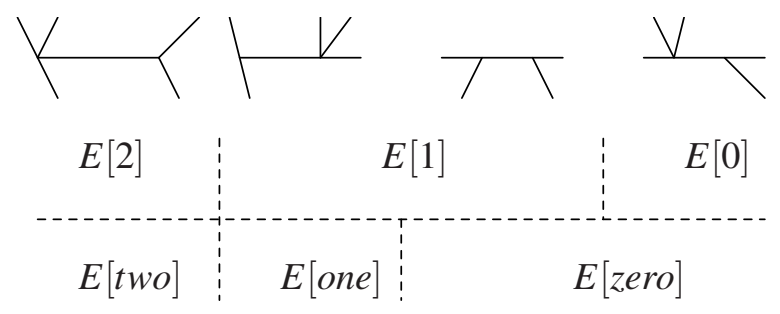

Fig. 4. In each picture, focus attention on the horizontal edge whose termini are both in view. This edge lies in the categories shown schematically below each picture. Both methods of classification are shown.

From the method stated above for the sampling of a typical edge-terminus, we have

$$
\begin{aligned}
\mathbb{P}_{E_{0}}\left\{e_{0} \in E_{0}[2]\right\}= & \mathbb{P}_{E}\{e \in E[\text { two }]\} \\
& \quad+\frac{1}{2} \mathbb{P}_{E}\{e \in E[\text { one }]\} \\
= & \varepsilon_{2}+\frac{1}{2} \mathbb{P}_{E}\{e \in E[\text { one }]\} .
\end{aligned}
$$

Using Eq. 22 and Eq. 23, we find that

$$
\begin{aligned}
& \varepsilon_{11}=\mathbb{P}_{E}\{e \in E[\text { one }]\}=2\left(1-\frac{2 \phi}{\theta}-\varepsilon_{2}\right), \\
& \varepsilon_{10}=\varepsilon_{1}-\varepsilon_{11}=\frac{4 \phi}{\theta}-\varepsilon_{1}-2 \varepsilon_{0} .
\end{aligned}
$$

For the general planar tessellation we see that, although we now have a finer classification of edges, no extra parameter is needed; $\varepsilon_{10}$ and $\varepsilon_{11}$ are not really new as they can be calculated from the parameters of our first classification. The finer classification does, however, play a role in some situations (as seen later in this section).

Linking $\varepsilon_{2}$ and $\phi$ : Eqs. 24 and 25 provide inequalities which link $\varepsilon_{2}$ and $\phi$. Writing Eq. 24 as $\varepsilon_{2}=1-2 \phi / \theta-\frac{1}{2} \varepsilon_{11}$, we can create an upper bound for $\varepsilon_{2}$ in terms of the ratio $\phi / \theta$. Similarly, Eq. 25 establishes a lower bound. These bounds are presented below in Eq. 26 and illustrated in Fig. 5 b.

$$
1-\frac{4 \phi}{\theta}+\varepsilon_{0} \leq \varepsilon_{2} \leq 1-\frac{2 \phi}{\theta} .
$$

Note that $\left(\varepsilon_{2}=1\right) \Longrightarrow(\phi=0)$ from the upper inequality and $(\phi=0) \Longrightarrow\left(\varepsilon_{2}=1\right)$ and $\left(\varepsilon_{0}=0\right)$ from the lower inequality and the fact that each epsilon $\in[0,1]$; so $(\phi=0)$ and $\left(\varepsilon_{2}=1\right)$ are equivalent, as is intuitively obvious.

The earlier examples revisited: In the STIT example, $\varepsilon_{11}=\frac{4}{3}(3-4 \log 2) \approx 0.3032$ whilst $\varepsilon_{10}=$ $\frac{2}{3}(2 \log 2-1)$ which happens to equal $\varepsilon_{0}$. Note also that $\bar{\ell}_{E[10]}=\bar{\ell}_{E[0]}$, proved by the methods in Cowan (2013), so therefore $\bar{\ell}_{E[11]}=\left(\varepsilon_{1} \bar{\ell}_{E[1]}-\varepsilon_{10} \bar{\ell}_{E[10]}\right) / \varepsilon_{11}=$ $3(3 \log 2-2) \bar{\ell}_{E} /(3-4 \log 2)=1.048 \bar{\ell}_{E}$.

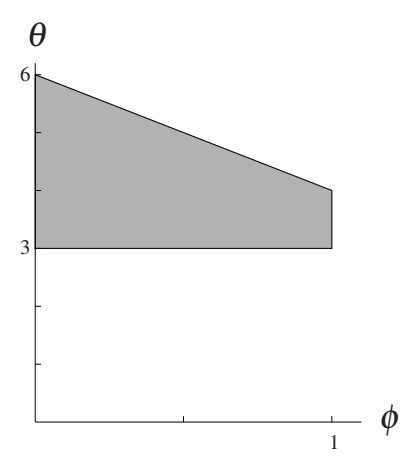

(a)

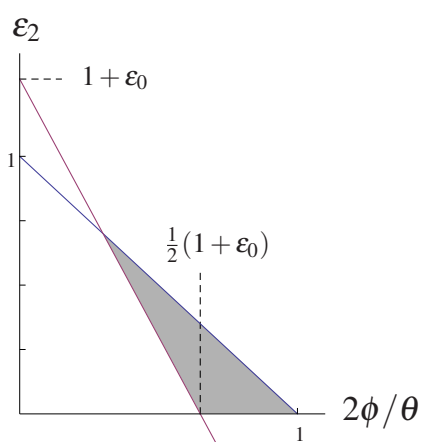

(b)
Fig. 5. (a) The permitted range for $(\phi, \theta)$ as described in the inequalities of $E q$. 1. (b) The permitted range for $\left(2 \phi / \theta, \varepsilon_{2}\right)$ for a given value of $\varepsilon_{0}$, taken from the inequality Eq. 26.

Note that $\varepsilon_{10}=0$ and $\varepsilon_{11}=\varepsilon_{1}=\frac{10}{21}$ in the Divided Delaunay tessellation $\mathscr{D}_{1}$, as expected from the description of that model (where type $E[10]$ is an impossibility).

In the $2 \times 1$-tiling, none of the $E[1]$-edges are of type $E[10]$, so $\varepsilon_{10}=0$ and $\varepsilon_{11}=\varepsilon_{1}=\frac{4}{9}$. Thus edges are either of type $E[2]$ or type $E[11]$, a feature that allows us later to compute the properties of this model combined in superposition with any isotropic model. Obviously, $\bar{\ell}_{E[11]}=\bar{\ell}_{E[1]}=1$.

The example in Fig. 6: An example which demonstrates $\varepsilon_{10}$ and $\varepsilon_{11}$ is seen in Fig. 6; it is described and partially analysed (yielding $\theta=\frac{19}{6}$ and $\phi=\frac{5}{6}$ ) in the figure's caption. The area of the shaded region is 32 so, because of the 7 cells, 19 edges and 12 vertices associated with this area, $\lambda_{Z}=\frac{7}{32}, \lambda_{E}=$ $\frac{19}{32}$ and $\lambda_{V}=\frac{3}{8}$. Furthermore, of the 19 edges, 4 are type $E[0], 10$ are $E[1]$ and 5 are $E[2]$. Therefore $\varepsilon_{0}=$ $\frac{4}{19}, \varepsilon_{1}=\frac{10}{19}$ and $\varepsilon_{2}=\frac{5}{19}$. Eq. 24 yields $\varepsilon_{11}=2\left(1-\frac{10}{19}-\right.$ $\left.\frac{5}{19}\right)=\frac{8}{19}$, so $\varepsilon_{10}=\frac{2}{19}$ from Eq. 25 . This agrees with the observed breakdown of type $E[1]$ into sub-types: 8 being $E[11]$ and 2 being $E[10]$.

Fig. 6 with each cell divided once: To illustrate the use of the classification of edges as shown in Fig. 4 , we consider the tessellation that arises when each cell of our rectangular tessellation (shown in Fig. 6) is independently divided by a chord - one which is uniformly random in the set of all vertical or horizontal chords of the cell, thus retaining rectangular cells. 


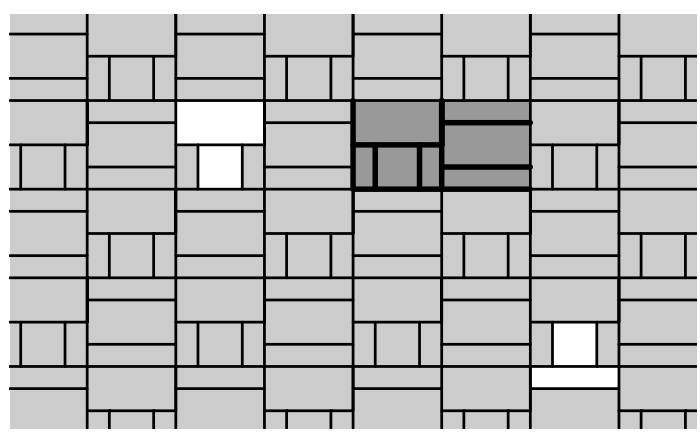

Fig. 6. A tessellation based on congruent copies of the region shown in dark shading arranged to cover the plane. It is made stationary by ensuring that the origin is uniformly distributed within the dark region. This region measures $8 \times 4$ and comprises 7 rectangular cells whose side lengths are of integer length, either 1, 2 or 4 units. The heavy lines within the dark region comprise 19 edges and there are 12 vertices within the region not counting those on its northern and eastern boundary (as these are counted in other copies of the region). Ten of the 12 vertices are $\pi$-vertices (each with 3 emanating edges) and the remaining two are not $\pi$ vertices (each having 4 emanating edges). So $\theta=\frac{19}{6}$ and $\phi=\frac{5}{6}$. The white patches, each containing two cells, are explained later in the text.

Consider firstly the top-left white patch; it contains a $4 \times 2$ upper cell and a $2 \times 2$ lower cell, separated by an edge of length 2 (which happens to be of type $E[10]$ ). Conditioning on the geometry of the patch, the chance that the separating edge is hit by the upper cell's chord is 2 times the edge's length divided by the perimeter of the upper cell, namely $\frac{1}{3}$. For the lower cell, the conditional chance is $\frac{1}{2}$. Given the geometry of the patch, these hitting events are independent. So the probability (given the patch geometry) that the separating edge is hit by 0,1 or 2 chords is $\frac{1}{3}, \frac{1}{2}$ and $\frac{1}{6}$ respectively. Repeating the calculation for the bottomright patch, where the separating edge is also of type $E[10]$, we get probabilities for 0,1 or 2 hits of $\frac{3}{10}, \frac{1}{2}$ and $\frac{1}{5}$ respectively, conditional on that patch's geometry. Any hit is independently uniformly distributed along the separating edge.

As these two patches (and their equally-prevalent copies throughout the plane) contain the only type $E[10]$ edges, we can say that the chance that a typical $E[10]$ edge receives 0,1 or 2 hits is $\frac{1}{2}\left(\frac{1}{3}+\frac{3}{10}\right)=\frac{19}{60}, \frac{1}{2}$ and $\frac{1}{2}\left(\frac{1}{6}+\frac{1}{5}\right)=\frac{11}{60}$ respectively. An $E[10]$ edge $e$ which is not hit remains of type $E[10]$ and hit twice becomes one $E[10]$ plus two $E[0]$ edges. Hit once by the chord in the cell whose side $s$ equals $e$, it becomes two $E[10]$ edges (the probability here is $\frac{19}{60}$ ). It becomes two $E[0]$ edges if the hit comes from the cell whose side $s \supset e$ (probability $\frac{11}{60}$ ).

The third row of Table 1 shows the resulting edgetypes produced from the possible hits on $E[10]$ edges.

The same exercise can be repeated for the eight edges that are of type $E[11]$. The post-division edgetypes differ from those in the $E[10]$ analysis (see Table 1 ), emphasising the need to break the type $E[1]$ into its two parts. The hit probabilities can be found for the typical $E[11]$ edge: no hits, $\frac{103}{180}$; two hits, $\frac{1}{18}$; one hit with $s=e, \frac{19}{90}$; one hit with $s \supset e, \frac{29}{180}$.

For the five $E[2]$ edges, Table 1 presents the resulting edge-types and a calculation shows that there will be 0,1 or 2 hits of the typical $E[2]$ edge with probabilities $\frac{8}{75}, \frac{11}{25}$ and $\frac{34}{75}$ respectively. The probabilities of 0,1 or 2 hits of the typical $E[0]$ edge are $\frac{5}{8}, \frac{1}{3}$ and $\frac{1}{24}$.

Table 1. Given the edge-type given in column one, the Table shows the types of edges that result from $j$ hits by chords, $j=0,1,2$. For example, a [0]-edge hit by 2 chords is subdivided into three edges; either all three will be type [0] or there will be two of type [10] and one of type [0]. Given $j=2$, the alternatives linked by the word 'or' are equally likely in all examples, but when $j=1$ the alternatives ' $s=e$ ' and ' $s \supset e$ ' will have example-dependent probabilities (see text).

\begin{tabular}{|c|c|c|c|}
\hline type & $j=0$ & $j=1$ & $j=2$ \\
\hline$[2]$ & {$[2]$} & $2[11]$ & $(2[11]+[0])$ \\
\hline$[11]$ & [11] & $\begin{array}{c}s=e \\
([11]+[10]) \\
\text { or } \\
s \supset e \\
([11]+[0])\end{array}$ & $\begin{array}{c}([11]+2[0]) \\
\text { or } \\
([11]+[10]+[0])\end{array}$ \\
\hline$[10]$ & [10] & $\begin{array}{cc}s=e & s \supset e \\
2[10] & \text { or } 2[0]\end{array}$ & $([10]+2[0])$ \\
\hline$[0]$ & {$[0]$} & $([10]+[0])$ & $\begin{array}{c}3[0] \\
\text { or } \\
(2[10]+[0])\end{array}$ \\
\hline
\end{tabular}

From these details and the information in Table 1, we can write down the intensity of the various edge types after all cells have been divided. Using * for the post-division results and no label for the pre-division terms, we have the following: 


$$
\begin{aligned}
& \lambda_{E[2]}^{*}= \frac{8}{75} \lambda_{E[2]}+\lambda_{Z}=\frac{113}{480} \\
& \lambda_{E[11]}^{*}=2\left(\frac{11}{25}+\frac{34}{75}\right) \lambda_{E[2]}+\lambda_{E[11]}=\frac{127}{240} \\
& \lambda_{E[10]}^{*}=\left(\frac{19}{90}+\frac{1}{2} \times \frac{1}{18}\right) \lambda_{E[11]}+\left(\frac{19}{60}+2 \times \frac{19}{60}+\frac{11}{60}\right) \lambda_{E[10]} \\
&+\left(\frac{1}{3}+\frac{1}{24}\right) \lambda_{E[0]}=\frac{511}{2880} \\
& \lambda_{E[0]}^{*}=\frac{34}{75} \lambda_{E[2]}+\left(\frac{29}{180}+\frac{1}{12}\right) \lambda_{E[11]}+2\left(\frac{11}{60}+\frac{11}{60}\right) \lambda_{E[10]} \\
&+\left(1+2 \times \frac{1}{48}\right) \lambda_{E[0]}=\frac{887}{2880} .
\end{aligned}
$$

Adding the four intensities above yields $\lambda_{E}^{*}=\frac{5}{4}$, a result which conforms with the simpler equation,

$$
\lambda_{E}^{*}=\lambda_{E}+3 \lambda_{Z}=\frac{19}{32}+3 \times \frac{7}{32}=\frac{5}{4} .
$$

Note that $\lambda_{E[1]}^{*}=\lambda_{E[11]}^{*}+\lambda_{E[10]}^{*}=\frac{407}{576}$. Also, the epsilons follow: firstly $\varepsilon_{2}^{*}=\lambda_{E[2]}^{*} / \lambda_{E}^{*}=\frac{113}{600}$, then similarly $\varepsilon_{1}^{*}=\frac{407}{720}$ and $\varepsilon_{0}^{*}=\frac{887}{3600}$. For completeness, $\lambda_{V}^{*}=\lambda_{V}+2 \lambda_{Z}=\frac{13}{16}$ and $\lambda_{V[\pi]}^{*}=\lambda_{V[\pi]}+2 \lambda_{Z}=\frac{3}{4}$, implying that $\theta^{*}=\frac{40}{13}$ and $\phi^{*}=\frac{12}{13}$.

REMARK 3: We note that the mathematics of this example is unchanged if, instead of using only horizontal and vertical chords, those which are distributed uniformly random (UR) on the set of all chords are used. The probability, given the cell's perimeter $b$, that a chord hits an edge of length $\ell$ is still $2 \ell / b$.

REMARK 4: Table 1 is universal; it holds for any tessellation and any mechanism for determining the dividing chords. So the point of our example is this: for any tessellation all four epsilons, $\varepsilon_{2}, \varepsilon_{0}, \varepsilon_{11}$ and $\varepsilon_{10}$ are essential inputs to the calculation of postdivision structure. Another input is also needed: the probabilities of 0,1 or 2 hits of the separating edge for each edge type. These probabilities were simply calculated in our example, but could be rather difficult to find in general.

The $\mathscr{D}_{2}$ iterate of the Delaunay: To illustrate Remark 4, we consider again the Divided Delaunay tessellation. We note that the first iterate $\mathscr{D}_{1}$ has no $E[10]$ edges, $\varepsilon_{10}=0$ being implied by the equality of $\varepsilon_{1}$ and $\varepsilon_{11}$. In the second iterate $\mathscr{D}_{2}$, however, we see from Table 1 that this type occurs $-E[10]$ can arise from the division of either $E[11], E[10]$ or $E[0]$ types. The relevant recurrences, after calculation of the hit probabilities and application of Table 1 (details omitted), are:

$$
\begin{aligned}
& \lambda_{E[2]}^{(2)}=\frac{4}{9} \lambda_{E[2]}^{(1)}+\lambda_{Z}^{(1)}=\frac{148}{27} \rho, \\
& \lambda_{E[11]}^{(2)}=\frac{10}{9} \lambda_{E[2]}^{(1)}+\lambda_{E[11]}^{(1)}=\frac{190}{27} \rho, \\
& \lambda_{E[10]}^{(2)}=\frac{11}{36} \lambda_{E[11]}^{(1)}+0 \times \lambda_{E[10]}^{(1)}+\frac{11}{36} \lambda_{E[0]}^{(1)}=\frac{121}{108} \rho, \\
& \lambda_{E[0]}^{(2)}=\frac{1}{9} \lambda_{E[2]}^{(1)}+\frac{7}{36} \lambda_{E[11]}^{(1)}+0 \times \lambda_{E[10]}^{(1)}+\frac{37}{36} \lambda_{E[0]}^{(1)}=\frac{49}{36} \rho .
\end{aligned}
$$

From these we get the basic epsilons for $\mathscr{D}_{2}: \varepsilon_{2}^{(2)}=$ $\frac{148}{405} ; \varepsilon_{1}^{(2)}=\frac{881}{1620} ; \varepsilon_{0}^{(2)}=\frac{49}{540}$. One can also show that $\theta_{\pi}^{(2)}=\frac{29}{9}$ and $\mu_{V E(2)}^{(2)}=\frac{262}{27}+\frac{256}{567} \times 37.7808 \approx 26.7617$. We do not present results for $\mathscr{D}_{n}, n>2$ as analysis of the hit probabilities becomes very complicated.

\section{EXAMPLES OF NESTING AND SUPERPOSITION}

These two operations which can be applied to a pair of tessellations are defined as follows.

Definition 6: Consider two tessellations with frames $\mathscr{Y}_{1}$ and $\mathscr{Y}_{2}$; the superposition is the tessellation with frame $\mathscr{Y}=\mathscr{Y}_{1} \cup \mathscr{Y}_{2}$. Object classes of $\mathscr{Y}$ are denoted by $V, E, Z, S, \ldots$, as usual, and those belonging to tessellation $\mathscr{Y}_{j}, j=1,2$, are denoted by $V^{(j)}, E^{(j)}, Z^{(j)}, S^{(j)}, \ldots$. A similar superscript is used for parameters of $\mathscr{Y}_{j}\left(\right.$ for example, $\left.\boldsymbol{\theta}^{(j)}, \phi^{(j)}, \ldots\right)$, except when such use is redundant (as in $\lambda_{E^{(j)}}^{(j)}$, whence either $\lambda_{E^{(j)}}$ or $\lambda_{E}^{(j)}$ suffices, the latter being easier to read).

In this section, $\mathscr{Y}_{1}$ and $\mathscr{Y}_{2}$ are assumed independent; an exception to this is given in the next section.

DEFINITION 7: To describe nesting, we again start with a tessellation frame $\mathscr{Y}_{1}$. Also we have available the distribution of the independent frame $\mathscr{Y}_{2}$, allowing us to produce independent replicates of $\mathscr{Y}_{2}$ when required. Then, for each closed cell $z$ of $\mathscr{Y}_{1}$, we independently generate a tessellation $\mathscr{Y}_{2}(z)$ distributed as $\mathscr{Y}_{2}$ and add $\mathscr{Y}_{2}(z) \cap z$ to $\mathscr{Y}_{1}$. Thus we create a tessellation $\mathscr{Y}$ equal to $\mathscr{Y}_{1}$ plus all 'nested components' inside the cells of $\mathscr{Y}_{1}$, that is, $\mathscr{Y}=\mathscr{Y}_{1} \cup \bigcup_{z}\left(\mathscr{Y}_{2}(z) \cap z\right)$ (Maier and Schmidt, 2003; Nagel and Weiss, 2003). Notation is the same as that in Definition 6, with the comment that the superscripted parameters and object classes when $j=2$ refer to $\mathscr{Y}_{2}$ and do not have a clear definition for $\mathscr{Y}_{2}(z) \cap z$ when $z \in Z^{(1)}$.

Many researchers have discussed the superposition and nesting of two tessellations, presenting formulae for parameters like $\lambda_{V}$ and $\lambda_{E}$ in the resulting process in terms of the parameters for each of the original tessellations (Santaló, 1984; Mecke, 1984; Weiss and Zähle, 1988; Maier and Schmidt, 2003; Nagel and Weiss, 2003; 2005). An assumption needed for reasonably pleasant formulae is that at least one of $\mathscr{Y}_{1}$ or $\mathscr{Y}_{2}$ should be isotropic. We too can reproduce those results very simply from Eq. 6 - and do so in the Appendix, taking $\mathscr{Y} / 2$ as the isotropic structure 
(an assumption which prevails in this section). The formulae that result are as follows, using $(\mathrm{S})$ and $(\mathrm{N})$ to mark the superposition and nesting cases respectively.

$$
\begin{aligned}
& \lambda_{E}=\left\{\begin{array}{l}
\lambda_{E}^{(1)}+\lambda_{E}^{(2)}+\frac{4}{\pi} \bar{\ell}_{E}^{(1)} \bar{\ell}_{E}^{(2)} \lambda_{E}^{(1)} \lambda_{E}^{(2)} \\
\lambda_{E}^{(1)}+\lambda_{E}^{(2)}+\frac{6}{\pi} \bar{\ell}_{E}^{(1)} \bar{\ell}_{E}^{(2)} \lambda_{E}^{(1)} \lambda_{E}^{(2)}
\end{array}\right. \\
& \lambda_{V}=\left\{\begin{array}{l}
\lambda_{V}^{(1)}+\lambda_{V}^{(2)}+\frac{2}{\pi} \bar{\ell}_{E}^{(1)} \bar{\ell}_{E}^{(2)} \lambda_{E}^{(1)} \lambda_{E}^{(2)} \\
\lambda_{V}^{(1)}+\lambda_{V}^{(2)}+\frac{4}{\pi} \bar{\ell}_{E}^{(1)} \bar{\ell}_{E}^{(2)} \lambda_{E}^{(1)} \lambda_{E}^{(2)}
\end{array}\right.
\end{aligned}
$$

We have a greater interest, however, in the effects of these operations on parameters like $\lambda_{V[\pi]}, \phi$ and $\varepsilon_{j}$. Santaló (1984), who first analysed the effects of superposition - and others cited above who extended his analysis and introduced nesting - were not concerned with these parameters. In the theory and examples given below and in the Appendix, we focus on $\lambda_{V[\pi]}, \phi$ and $\varepsilon_{j}, j=0,1,2$, while drawing on the known results Eq. 27 and Eq. 28.

The parameters $\lambda_{V[\pi]}$ and $\phi$ : For superpositions it is easily seen that the only $\pi$-vertices in $\mathscr{Y}$ are those that were $\pi$-vertices in the initial tessellations $\mathscr{Y}_{1}$ and $\mathscr{Y}_{2}$; no new $\pi$-vertices are created by the intersection of the two frames. On the other hand, nesting produces new $\pi$-vertices created by the intersection of $\mathscr{Y}_{1}$ with $\bigcup_{z}\left(\mathscr{Y}_{2}(z) \cap z\right)$ whilst also retaining the original $\pi$ vertices of $\mathscr{Y}_{1}$ and of the nested replicates of $\mathscr{Y}_{2}$. These remarks on $\pi$-vertices lead (with the help of Eq. 31 in the Appendix) to the following formulae.

$$
\lambda_{V[\pi]}=\left\{\begin{array}{l}
\lambda_{V[\pi]}^{(1)}+\lambda_{V[\pi]}^{(2)} \\
\lambda_{V[\pi]}^{(1)}+\lambda_{V[\pi]}^{(2)}+\frac{4}{\pi} \bar{\ell}_{E}^{(1)} \bar{\ell}_{E}^{(2)} \lambda_{E}^{(1)} \lambda_{E}^{(2)}(\mathrm{N})
\end{array}\right.
$$

The parameter $\phi$ is given by $\lambda_{V[\pi]} / \lambda_{V}$.

Edge-types: In both contexts, we say that an edge in the resulting tessellation frame $\mathscr{Y}$ is of $\mathscr{Y}_{1}$-genesis if it is a subset of the $\mathscr{Y}_{1}$ frame. Otherwise, it is of $\mathscr{Y}_{2}$-genesis. It is important to note that an edge of $\mathscr{Y}_{i}{ }^{-}$ genesis might not be an edge of $\mathscr{Y}_{i}$, but only a subset of one.

REMARK 5: If $\mathscr{Y}_{2}$ is side-to-side, all of the edges in $\mathscr{Y}$ of $\mathscr{Y}_{2}$-genesis will be of type E[2], whether $\mathscr{Y}$ is a superposition or a nesting. If $\mathscr{Y}_{2}$ is not side-to-side, results about edge-types are usually less amenable.

A superposition example: The $(2 \times 1)$-tiling seen above plays the role of $\mathscr{Y}_{1}$. Its superposition partner $\mathscr{Y}_{2}$ is our isotropic Delaunay tessellation $\mathscr{D}_{0}$, chosen to have parameter $\rho=(3 \pi / 8)^{2} \approx 1.388$ so that $\lambda_{E}^{(1)}=$
$\lambda_{E}^{(2)}$ in conformity with Fig. 7. Therefore, in this example, $\mathscr{Y}_{2}$ is side-to-side and $\mathscr{Y}_{1}$ has no edges of types $E[10]$ or $E[0]$, a feature which enables an easy analysis. From this earlier analysis, we know that

$$
\begin{array}{lll}
\lambda_{V}^{(1)}=\frac{5}{8}, & \lambda_{E}^{(1)}=\frac{9}{8}, & \theta^{(1)}=\frac{18}{5}, \\
\lambda_{V}^{(2)}=\frac{9 \pi^{2}}{64}, & \lambda_{E}^{(2)}=\frac{27 \pi^{2}}{64}, & \theta^{(2)}=6,
\end{array}
$$

and

$$
\begin{array}{lll}
\bar{\ell}_{E}^{(1)}=\frac{4}{3}, & \lambda_{V[\pi]}^{(1)}=\frac{1}{4}, & \phi^{(1)}=\frac{2}{5}, \\
\bar{\ell}_{E}^{(2)}=\frac{4}{3}, & \lambda_{V[\pi]}^{(2)}=0, & \phi^{(2)}=0 .
\end{array}
$$

So, from Eqs. 27-29,

$$
\begin{aligned}
\lambda_{V} & =\frac{1}{64}\left(40+108 \pi+9 \pi^{2}\right) \approx 7.3, & \lambda_{V[\pi]} & =\frac{1}{4}, \\
\theta & =2 \frac{\lambda_{E}}{\lambda_{V}} \approx 4.35, & \phi=\frac{\lambda_{V[\pi]}}{\lambda_{V}} & \approx 0.034, \\
\lambda_{E} & =\frac{9}{64}\left(8+24 \pi+3 \pi^{2}\right) \approx 15.9, & \bar{\ell}_{E} & =\frac{4}{3} .
\end{aligned}
$$

Also $\lambda_{E[2]}^{(1)}=\frac{5}{8}, \lambda_{E[11]}^{(1)}=\frac{1}{2}, \bar{\ell}_{E[2]}^{(1)}=\frac{8}{5}$ and $\bar{\ell}_{E[11]}^{(1)}=$ 1 , from the earlier calculations. Thus, because every type $E[11]$ in $\mathscr{Y}_{1}$, whether remaining intact or not, yields exactly one $E[11]$ for $\mathscr{Y}$ (and there are no $E[11]$-type edges of $\mathscr{Y}_{2}$-genesis because $\mathscr{Y}_{2}$ is sideto-side), $\lambda_{E[11]}=\frac{1}{2}$. Therefore $\lambda_{E[2]}=\lambda_{E}-\lambda_{E[11]}=$ $\frac{5}{8}+\frac{27}{64} \pi(\pi+8) \approx 15.39$. As Fig. 7 suggests, edge type $E[2]$ now dominates - as reflected in the epsilon parameters: $\varepsilon_{2}=1-\frac{32}{9}\left(8+24 \pi+3 \pi^{2}\right)^{-1} \approx$ $0.97 ; \varepsilon_{11}=\varepsilon_{1} \approx 0.03 ; \varepsilon_{10}=\varepsilon_{0}=0$. These calculations are in agreement with the more general treatment of superpositions given in the Appendix.

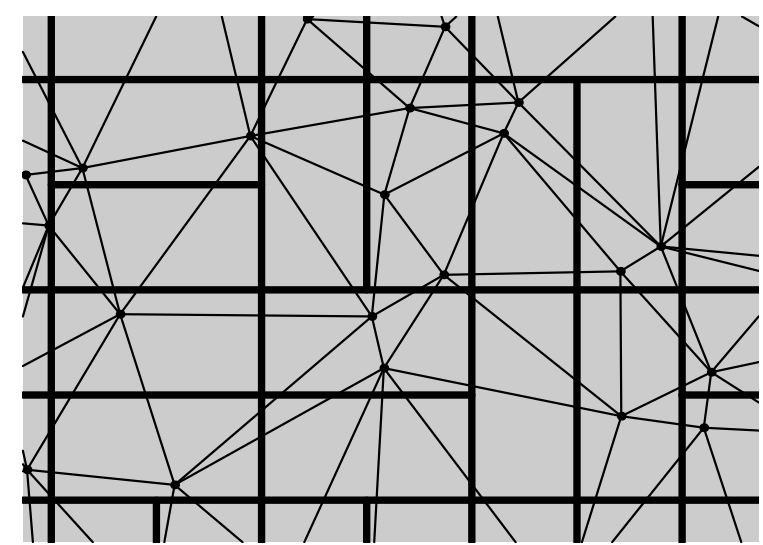

Fig. 7. The bold line-segments form the frame of a $2 \times 1$ tiling. Superimposed on it is our Delaunay tessellation $\mathscr{D}$. Parameters have been chosen so the mean edge lengths in the two tessellations are equal. 
A nesting example: Here both $\mathscr{Y}_{1}$ and $\mathscr{Y} / 2$ are isotropic Poisson line processes, having 'point processes on line-transects' with intensities $\rho^{(1)}$ and $\rho^{(2)}$ respectively. These point processes are Poisson processes, so the tessellations have the Poisson Transect Property discussed in Remark 1. Also the typical edge in either tessellation has a length that is exponentially distributed. The relevant parameters of $\mathscr{Y}_{1}$ and $\mathscr{Y}_{2}$, calculated in Miles (1973) are as follows:

$$
\begin{gathered}
\lambda_{V}^{(1)}=\frac{\pi}{4}\left(\rho^{(1)}\right)^{2}, \quad \lambda_{E}^{(1)}=\frac{\pi}{2}\left(\rho^{(1)}\right)^{2}, \quad \theta^{(1)}=4, \\
\lambda_{V}^{(2)}=\frac{\pi}{4}\left(\rho^{(2)}\right)^{2}, \quad \lambda_{E}^{(2)}=\frac{\pi}{2}\left(\rho^{(2)}\right)^{2}, \quad \theta^{(2)}=4, \\
\bar{\ell}_{E}^{(1)}=\frac{1}{\rho^{(1)}}, \quad \lambda_{V[\pi]}^{(1)}=0, \quad \phi^{(1)}=0, \\
\bar{\ell}_{E}^{(2)}=\frac{1}{\rho^{(2)}}, \quad \lambda_{V[\pi]}^{(2)}=0, \quad \phi^{(2)}=0 .
\end{gathered}
$$

From these, and using Eqs. 27-29 together with Lemma 3 and Eq. 32, we calculate

$$
\begin{aligned}
\lambda_{V} & =\frac{\pi}{4}\left(\left(\rho^{(1)}+\rho^{(2)}\right)^{2}+2 \rho^{(1)} \rho^{(2)}\right), \\
\lambda_{E} & =\frac{\pi}{2}\left(\left(\rho^{(1)}+\rho^{(2)}\right)^{2}+\rho^{(1)} \rho^{(2)}\right), \\
\lambda_{V[\pi]} & =\frac{\pi}{2} \rho^{(1)} \rho^{(2)}, \phi=\frac{\lambda_{V[\pi]}}{\lambda_{V}} \quad \text { and } \quad \theta=2 \frac{\lambda_{E}}{\lambda_{V}} .
\end{aligned}
$$

Using Lemma 3, Eq. 32 and Eq. 33, we find that

$$
\begin{array}{ll}
\lambda_{E\left[\mathscr{V}_{1}\right]}=\frac{\pi}{2} \rho^{(1)}\left(\rho^{(1)}+2 \rho^{(2)}\right), \bar{\ell}_{E\left[\mathscr{V}_{1}\right]}=\frac{1}{\rho^{(1)}+2 \rho^{(2)}}, \\
\lambda_{E\left[\mathscr{G}_{2}\right]}=\frac{\pi}{2} \rho^{(2)}\left(\rho^{(1)}+\rho^{(2)}\right), & \bar{\ell}_{E\left[\mathscr{G}_{2}\right]}=\frac{1}{\rho^{(1)}+\rho^{(2)}},
\end{array}
$$

and

$$
\bar{\ell}_{E}=\frac{\rho^{(1)}+\rho^{(2)}}{\left(\rho^{(1)}+\rho^{(2)}\right)^{2}+\rho^{(1)} \rho^{(2)}} .
$$

Fig. 8 shows $\mathscr{Y} / 1$ and, inside two neighbouring cells $z$ and $z *$ of $\mathscr{Y}_{1}$, the nesting by $\mathscr{Y}_{2}$. We note that all edges of $\mathscr{Y}_{2}$-genesis contained in $z$ (or in $z *$ ) are of type $E[2]$. The figure also shows the line-segment $z \cap z *$ which was originally an edge (e say) of $\mathscr{Y}_{1}$. Postnesting, it has been transformed into five edges of $\mathscr{Y}_{1}$ genesis, but in general the types of these edges may be $E[11], E[10]$ or $E[0]$ depending on the positions of the hits of $e=z \cap z *-$ and whether the hits are caused by chords of $z$ or chords of $z *$.

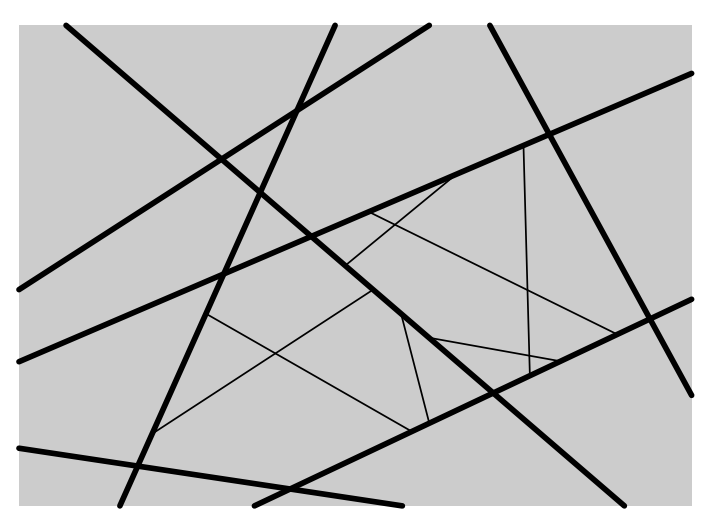

Fig. 8. The bold lines show the frame $\mathscr{Y}_{1}$ of a line process into which independent copies of a line process $\mathscr{Y}_{2}$ will be nested. In order to focus attention on two neighbouring cells $z$ and $z *$ of $\mathscr{Y}_{1}$ and the five postnesting edges of $\mathscr{Y}_{1}$-genesis on the line-segment $z \cap z *$, we only show the nesting in these two cells of $\mathscr{Y}_{1}$. Inside these cells, we see the post-nesting edges of $\mathscr{Y}_{2}-$ genesis as thin lines.

Because $\mathscr{Y}_{2}$ has the Poisson Transect Property, $\mathrm{K}_{e}$ has the Poisson distribution with mean $2 \rho^{(2)} \ell$, given the length $\ell$ of an edge $e$ of $\mathscr{Y}_{1}$. Moreover, given $\ell$ and given $\mathrm{K}_{e}=k>0$, these $k$ hits are uniformly and independently positioned on the edge $e$ and equally likely to be caused by chords in $z$ or $z *$. Thus, the $k$ hits create two $E[11]$ edges, an expected $(k-1) / 2$ edges of $E[10]$ type and also an expected $(k-1) / 2$ edges of $E[0]$ type. In Fig. 8, $k=4$ and we see two $E[0]-$ edges and one $E[10]$-edge, along with the two $E[11]$ type edges. When $k=0$, the edge remains type $E[2]$. Therefore, the typical edge of $\mathscr{Y}_{1}$ (in this example all edges of $\mathscr{Y}_{1}$ are type $E[2]$ ) has an expected number of

$$
\int_{0}^{\infty} \sum_{k \geq 1} \frac{k-1}{2} \mathbb{P}_{E^{(1)}}\left\{\mathrm{K}_{e}=k \mid \ell\right\} f(\ell) \mathrm{d} \ell
$$

type $-E[0]$ edges. Here, $f$ is the probability density function of $e$ 's length $\ell$, known to be $\rho^{(1)} \exp \left(-\rho^{(1)} \ell\right)$ in this example because $\mathscr{Y}_{1}$ is also a Poisson line process. Evaluating the formula above, we get

$$
\begin{array}{r}
\int_{0}^{\infty} \sum_{k \geq 1} \frac{k-1}{2} \frac{\left(2 \rho^{(2)} \ell\right)^{k} \mathrm{e}^{-2 \rho^{(2)} \ell}}{k !} \rho^{(1)} \mathrm{e}^{-\rho^{(1)} \ell} \mathrm{d} \ell \\
=\frac{1}{2} \int_{0}^{\infty}\left(\mathrm{e}^{-2 \rho^{(2)} \ell}-1+2 \rho^{(2)} \ell\right) \rho^{(1)} \mathrm{e}^{-\rho^{(1)} \ell} \mathrm{d} \ell \\
=\frac{2\left(\rho^{(2)}\right)^{2}}{\rho^{(1)}\left(\rho^{(1)}+2 \rho^{(2)}\right)} .
\end{array}
$$

An identical argument and result applies for $E[10]$-type edges. Therefore the intensities of $E[0]$-type and $E[10]$ type edges are given by 


$$
\begin{aligned}
\lambda_{E[0]}=\lambda_{E[10]} & =\lambda_{E}^{(1)} \frac{2\left(\rho^{(2)}\right)^{2}}{\rho^{(1)}\left(\rho^{(1)}+2 \rho^{(2)}\right)} \\
& =\frac{\pi \rho^{(1)}\left(\rho^{(2)}\right)^{2}}{\rho^{(1)}+2 \rho^{(2)}} .
\end{aligned}
$$

A similar method yields

$$
\lambda_{E[11]}=\frac{2 \pi\left(\rho^{(1)}\right)^{2} \rho^{(2)}}{\rho^{(1)}+2 \rho^{(2)}}
$$

and this provides

$$
\lambda_{E[1]}=\lambda_{E[11]}+\lambda_{E[10]}=\frac{\pi \rho^{(1)} \rho^{(2)}\left(2 \rho^{(1)}+\rho^{(2)}\right)}{\rho^{(1)}+2 \rho^{(2)}} .
$$

After tallying both the edges of $\mathscr{Y}_{2}$-genesis and those edges from $\mathscr{Y}_{1}$ which have not been hit, we have

$$
\begin{aligned}
\lambda_{E[2]} & =\lambda_{E\left[\mathscr{V}_{2}\right]}+\frac{\pi\left(\rho^{(1)}\right)^{3}}{2\left(\rho^{(1)}+2 \rho^{(2)}\right)} \\
& =\frac{\pi}{2} \rho^{(2)}\left(\rho^{(1)}+\rho^{(2)}\right)+\frac{\pi\left(\rho^{(1)}\right)^{3}}{2\left(\rho^{(1)}+2 \rho^{(2)}\right)} .
\end{aligned}
$$

A check shows that $\lambda_{E[0]}+\lambda_{E[1]}+\lambda_{E[2]}=\lambda_{E}$. Finally one is able to calculate $\varepsilon_{j}=\lambda_{E[j]} / \lambda_{E}$ and for the case illustrated in Fig. 8, where $\rho^{(2)}=2 \rho^{(1)}$, the calculation yields $\left\{\varepsilon_{0}, \varepsilon_{1}, \varepsilon_{2}\right\}=\left\{\frac{8}{55}, \frac{16}{55}, \frac{31}{55}\right\}$.

\section{CONCLUSION}

What have we achieved in this paper that cannot be found in the standard text of Stoyan et al. (1995)? By drawing on the primary sources (Cowan, 1978; 1980; Mecke, 1980), the authors of this text presented formulae involving the three intensities $\lambda_{V}, \lambda_{E}$ and $\lambda_{Z}$ and the nine adjacencies $\mu_{X Y}$ where $X, Y \in\{V, E, Z\}$, one of these adjacencies being equivalent to our $\theta$. Stoyan et al. demonstrated that all of these entities can be expressed as functions of $\lambda_{V}$ and $\theta$. They also noted that the metric entities, $\bar{a}_{Z}, \bar{\ell}_{Z}$ and frame intensity, are functions of the mean edge length $\bar{\ell}_{E}$ (and of $\lambda_{V}$ and $\theta)$.

Their list of results, which has influenced quite a number of studies and other texts, applies to all stationary planar tessellations whether side-to-side or not. The problem is that, when a tessellation is not side-to-side, their results only tell part of the story: just that part which involves the primitive tessellation elements vertices, edges and cells. An interesting and important part of the story, that involving faces of the primitive elements such as the sides of polygonal cells - and the distinction between sides and edges - is not illuminated by their list of results.
Our paper tells much more of this story. To explain what is happening with the lower-dimensional faces of edges $E$ and cells $Z$, we have introduced (via Definition 4) the four extra object classes $E_{0}, S, C$ and $S_{0}$ and utilised the extra parameters $\phi, \mu_{V E(2)}, \theta_{\pi}$ and $\varepsilon_{j}, j=0,1,2$. Thus we study the seven classes of object $\left\{V, E, Z, E_{0}, C, S, S_{0}\right\}$ compared to the three classes $\{V, E, Z\}$ studied in Stoyan et al. (1995). We draw upon the incomplete $7 \times 7$ table of adjacency results in Weiss and Cowan (2011), a table which we complete in Theorem 1 by employing the three parameters $\varepsilon_{1}, \varepsilon_{2}$ and $\varepsilon_{3}$. These three epsilons, although not the only parameters we introduce to describe non side-to-side features, play the central role in our characterisation of tessellations which are not side-to-side. We suggest that they capture the essence of the non side-to-side structure. Many examples, some interesting in their own right and others mainly for reinforcement of the ideas, round out the theories.

\section{ACKNOWLEDGEMENT}

We thank the reviewers for their careful reading of the manuscript and for their suggestions.

After this paper was submitted for publication, a third edition of the text by Stoyan et al appeared (see Chiu et al. (2013)). That edition embraces many of the ideas and notations of Weiss and Cowan (2011). The text now makes use of the parameter $\phi$ from Cowan (1978), and presents a distinction between sides and edges. Nevertheless, our paper deals with these issues in much greater detail, through our introduction of the parameters $\varepsilon_{1}, \varepsilon_{2}$ and $\varepsilon_{3}$.

\section{REFERENCES}

Chui SN, Stoyan D, Kendall WS, Mecke J (2013). Stochastic Geometry and its Applications. 3rd Ed. Chichester: Wiley.

Cowan R (1978). The use of ergodic theorems in random geometry. Suppl Adv Appl Prob 10:47-57.

Cowan R (1979). Homogeneous line-segment processes. Math Proc Camb Phil Soc 86:481-9.

Cowan R (1980). Properties of ergodic random mosaic processes. Math Nachr 97:89-102.

Cowan R, Morris VB (1988). Division rules for polygonal cells. J Theor Biol 131:33-42.

Cowan R (2004). A mosaic of triangular cells formed with sequential splitting rules. J Appl Prob Spc Vol 41A:3-15.

Cowan R (2010). New classes of random tessellations arising from iterative division of cells. Adv Appl Prob 42:26-47. 
Cowan R (2013). Line-segments in the isotropic planar STIT tessellation. Adv Appl Prob 45:295-311.

Cowan R, Tsang AKL (1994). The falling-leaf mosaic and its equilibrium properties. Adv Appl Prob 26:54-62.

Grünbaum B, Shephard GC (1987). Tilings and Patterns. New York: WH Freeman.

Heinrich L, Muche L (2008). Second-order properties of the point process of nodes in a stationary Voronoi tessellation. Math Nachr 281:350-75.

Leistritz L, Zähle M (1992). Topological mean value relationships for random cell complexes. Math Nachr 155:57-72.

Maier R, Schmidt V (2003). Stationary iterated tessellations. Adv Appl Prob 35:337-53.

Mecke J (1980). Palm methods for stationary random mosaics. In: Ambartzumian RA, Ed. Combinatorial Principles in Stochastic Geometry. Erevan: Armenian Academy of Science.

Mecke J (1984). Parametric representation of mean values for stationary random mosaics. Math Operations Statist Ser Statist 15:437-42.

Mecke J, Nagel W, Weiss V (2007). Length distributions of edges in planar stationary and isotropic STIT tessellations. Izvest Akad Nauk Armen Matem 42:3960.

Mecke J, Nagel W, Weiss V (2011). Some distributions for I-segments of planar random homogeneous STIT tessellations. Math Nachr 284:1483-95.

Miles RE (1970). On the homogeneous planar Poisson point process. Math Biosci 6:85-127.

Miles RE (1973). The various aggregates of random polygons determined by random lines in a plane. Adv Math 10:256-90.

Møller J (1989). Random tessellations in $\mathrm{R}^{d}$. Adv Appl Prob 21:37-73.

Nagel W, Weiss V (2003). Limits of sequences of stationary planar tessellations. Adv Appl Prob 35:123-38.

Nagel W, Weiss V (2005). Crack STIT tessellations: characterization of stationary random tessellations stable with respect to iteration. Adv Appl Prob 37:859-83.

Santaló LA (1984). Mixed random mosaics. Math Nachr 117:129-33.

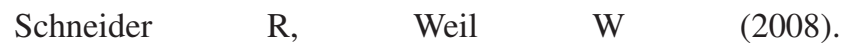
Stochastic and Integral Geometry. Berlin Heidelberg: Springer.

Schreiber T, Thäle C (2010). Second-order properties and central limit theory for the vertex process of iteration infinitely divisible and iteration stable random tessellations in the plane. Adv Appl Prob 42:913-35.

Schreiber T, Thäle C (2013). Limit theorems for iteration stable tessellations. Ann Prob 41:2261-78.

Stoyan D, Kendall WS, Mecke J (1995). Stochastic Geometry and its Applications. 2nd Ed. Chichester: Wiley. .

Weiss V, Cowan R (2011). Topological relationships in spatial tessellations. Adv Appl Prob 43:963-84.

Weiss V, Zähle M (1988). Geometric measures for random curved mosiacs of $\mathrm{R}^{d}$. Math Nachr 138:313-26.

\section{APPENDIX: SUPERPOSITION AND NESTING FORMULAE}

This Appendix links our formulae, Eqs. 6-8, with superposition and nesting. It supplements the section where these two tessellation operations and related terminology are defined, and should be read in parallel with it.

Assuming that $\mathscr{Y} / 2$ is isotropic, we ask what is the expected number of $\mathscr{Y}_{2}$-genesis edges in $\mathscr{Y}$ contained in the typical cell of the $\mathscr{Y}_{1}$ tessellation? To answer this question, we simply condition on the area and perimeter of a $\mathscr{Y}_{1}$-genesis cell $z$, apply Eq. 6 (with $W=z$ and using $\bar{\ell}_{E}^{(2)}$ and $\lambda_{E}^{(2)}$ for the $\bar{\ell}_{E}$ and $\lambda_{E}$ in this formula) and take the expectation $\mathbb{E}_{Z^{(1)}}$ of the conditioned entities. This gives (in both the superposition and nesting contexts) an expected number of edges of $\mathscr{Y}_{2}$-genesis contained in a typical cell of tessellation $\mathscr{Y}_{1}$ as

$$
\mathbb{E}_{Z^{(1)}}\left(\mathrm{K}_{z}\left(E^{(2)}\right)\right)=\left(\frac{\bar{\ell}_{E}^{(2)}}{\pi} \bar{\ell}_{Z}^{(1)}+\bar{a}_{Z}^{(1)}\right) \lambda_{E}^{(2)} .
$$

Using Eq. 30, the intensity of edges of $\mathscr{Y}_{2}$ genesis is $\lambda_{Z}^{(1)}\left(\bar{\ell}_{E}^{(2)} \bar{\ell}_{Z}^{(1)} / \pi+\bar{a}_{Z}^{(1)}\right) \lambda_{E}^{(2)}$ which equals $\lambda_{E}^{(2)}\left(1+2 \bar{\ell}_{E}^{(1)} \bar{\ell}_{E}^{(2)} \lambda_{E}^{(1)} / \pi\right)$ from Eq. 2 and Eq. 4.

REMARK 7: In Eq. 30, we use $\mathbb{E}_{Z^{(1)}}(\cdot)$ to indicate that the 'typicality expectation' is taken for cells $z \in$ $Z^{(1)}$, that is, only cells of $\mathscr{Y}_{1}$. We have used $E^{(2)}$ for the argument of $\mathrm{K}_{z}$ to indicate that we count only the edges of $\mathscr{Y}_{2}$. In this Appendix, however, edges are the only objects of $\mathscr{Y}_{2}$ that we count - we don't count other entities such as vertices or cells - and so, for brevity, we drop entirely the bracketed argument of $\mathrm{K}$ that was a necessary part of Eqs. 6-8. We retain only the subscript argument, so the left-hand part of Eq. 30 simplifies to $\mathbb{E}_{Z^{(1)}} \mathrm{K}_{z}$.

Using Eq. 8 similarly, the expected number of edges of $\mathscr{Y}_{2}$-genesis hitting the typical type- $X$ edge of $\mathscr{Y}_{1}$ is

$$
\mathbb{E}_{X^{(1)}} \mathrm{K}_{x}=\frac{2}{\pi} \bar{\ell}_{X}^{(1)} \bar{\ell}_{E}^{(2)} \lambda_{E}^{(2)}
$$


for the superposition and double this value for the nested tessellation due to independent hits of the edge from both adjacent cells (see Fig. 8 for illustration). Here $x \in X$. In the superposition case, all of these hits create $\bar{\pi}$-vertices (of order 4 ) on this edge of $\mathscr{Y}_{1}$; in the nesting case, all the hits are $\pi$-vertices (of order 3 ).

Therefore the intensity of $\mathscr{Y}_{1}$-genesis edges in $\mathscr{Y}$ is $\lambda_{E}^{(1)}\left(1+\mathbb{E}_{E^{(1)}} \mathrm{K}_{e}\right)$ which can be evaluated from Eq. 31 using $X=E^{(1)}$. The overall edge intensity in $\mathscr{Y}$ is that given in Eq. 27, in agreement with formulae (4.6) and (4.17) of Maier and Schmidt (2003). Moreover, accounting for the new vertices created whose intensity is given by Eq. 31, the overall intensity $\lambda_{V}$ of vertices is as per Eq. 28, in agreement with formulae (4.5) and (4.16) of Maier and Schmidt.

Thus we have proved the following result, presented here as a Lemma.

Lemma 3: Let $E\left[\mathscr{Y}_{j}\right]$ denote the class of $\mathscr{Y}_{j^{-}}$ genesis edges. The intensity of edges of $\mathscr{Y}_{2}$-genesis is

$$
\lambda_{E\left[\mathscr{V}_{2}\right]}=\lambda_{E}^{(2)}\left(1+\frac{2}{\pi} \bar{\ell}_{E}^{(1)} \bar{\ell}_{E}^{(2)} \lambda_{E}^{(1)}\right),
$$

both for superpositions and nestings. The intensity of edges of $\mathscr{Y}_{1}$-genesis equals $\lambda_{E}-\lambda_{E\left[\mathscr{Y}_{2}\right]}$, or

$$
\lambda_{E\left[\mathscr{Y}_{1}\right]}=\left\{\begin{array}{l}
\lambda_{E}^{(1)}\left(1+\frac{2}{\pi} \bar{\ell}_{E}^{(1)} \bar{\ell}_{E}^{(2)} \lambda_{E}^{(2)}\right), \\
\lambda_{E}^{(1)}\left(1+\frac{4}{\pi} \bar{\ell}_{E}^{(1)} \bar{\ell}_{E}^{(2)} \lambda_{E}^{(2)}\right),
\end{array}\right.
$$

The proportion of edges which are of $\mathscr{Y}_{j}$-genesis equals $\lambda_{E\left[\mathscr{Y}_{j}\right]} / \lambda_{E}$.

Moreover, in both contexts, the frame intensity of $\mathscr{Y}$ equals the sum of frame intensities for $\mathscr{Y}_{1}$ and $\mathscr{Y}_{2}$. This means that $\bar{\ell}_{E} \lambda_{E}=\bar{\ell}_{E}^{(1)} \lambda_{E}^{(1)}+\bar{\ell}_{E}^{(2)} \lambda_{E}^{(2)}$; so the mean edge length of $\mathscr{Y}$ is as follows:

$$
\bar{\ell}_{E}=\frac{\bar{\ell}_{E}^{(1)} \lambda_{E}^{(1)}+\bar{\ell}_{E}^{(2)} \lambda_{E}^{(2)}}{\lambda_{E}}
$$

It is also true that $\bar{\ell}_{E\left[\mathscr{Y}_{j}\right]} \lambda_{E\left[\mathscr{Y}_{j}\right]}$ equals the frame intensity of $\mathscr{Y}_{j}$, as does $\bar{\ell}_{E}^{(j)} \lambda_{E}^{(j)}$. So

$$
\bar{\ell}_{E\left[\mathscr{Y}_{j}\right]}=\frac{\bar{\ell}_{E}^{(j)} \lambda_{E}^{(j)}}{\lambda_{E\left[\mathscr{Y}_{j}\right]}}, \quad j=1,2 .
$$

Further formulae, relevant to our study and proved with similar accountancy, are given in the main section on superposition and nesting.

The tessellation $\mathscr{Y}_{2}$ need not be isotropic for these formulae above to hold. If it is $\mathscr{Y}_{1}$ that is isotropic and $\mathscr{Y} / 2$ not, then the formulae still hold (though the argument is more elaborate and works because the typical cell and typical edge of $\mathscr{Y}_{1}$ would then be isotropic random sets).
Edge-types formed with superposition: With superpositions, the number of hits by the edges of $\mathscr{Y}_{2}$ on a typical edge $x$ in $\mathscr{Y}_{1}$ of type $X$ is denoted by $\mathrm{K}_{x}$. It has expectation given in Eq. 31 . The $\mathrm{K}_{x}$ hits partition the original type- $X$ edge into $\mathrm{K}_{x}+1$ edges. When $X=E[11]$, one of the created edges is $E[11]$ and the remainder are $E[2]$. When $X=E[2]$, all of the created edges are of type $E[2]$.

The situations when $X=E[10]$ or $X=E[0]$ edges are more complicated, because the $\mathrm{K}_{x}+1$ resulting edges comprise two of type $E[11]$ and $\left(\mathrm{K}_{x}-1\right)$ of type $E[2]$ when $\mathrm{K}_{x}>0$. When $\mathrm{K}_{x}=0$, the edge type is unchanged. So, the intensities of edges in $\mathscr{Y}$ are given below, where the $e$ in terms like $\mathbb{P}_{E[\cdot]^{(1)}}\left\{\mathrm{K}_{e}=0\right\}$ or $\mathbb{E}_{E[\cdot]^{(1)}} \mathrm{K}_{e}$ refers generically to an edge in $E[\cdot]^{(1)}$ (the subscript of the Palm measure or Palm expectation applying to that term).

$$
\begin{aligned}
\lambda_{E[10]}= & \lambda_{E[10]}^{(1)} \mathbb{P}_{E[10]^{(1)}}\left\{\mathrm{K}_{e}=0\right\}, \\
\lambda_{E[0]}= & \lambda_{E[0]}^{(1)} \mathbb{P}_{E[0]^{(1)}}\left\{\mathrm{K}_{e}=0\right\}, \\
\lambda_{E[11]}= & \lambda_{E[11]}^{(1)}+2 \lambda_{E[10]}^{(1)} \mathbb{P}_{E[10]^{(1)}}\left\{\mathrm{K}_{e}>0\right\} \\
& +2 \lambda_{E[0]}^{(1)} \mathbb{P}_{E[0]^{(1)}}\left\{\mathrm{K}_{e}>0\right\},
\end{aligned}
$$

and

$$
\begin{aligned}
\lambda_{E[2]}= & \lambda_{E}^{(2)}\left(1+\frac{2}{\pi} \bar{\ell}_{E}^{(1)} \bar{\ell}_{E}^{(2)} \lambda_{E}^{(2)}\right) \\
& +\lambda_{E[2]}^{(1)}\left(1+\mathbb{E}_{E[2]^{(1)}} \mathrm{K}_{e}\right)+\lambda_{E[11]}^{(1)} \mathbb{E}_{E[11]^{(1)}} \mathrm{K}_{e} \\
& +\lambda_{E[10]}^{(1)}\left(\mathbb{E}_{E[10]^{(1)}} \mathrm{K}_{e}-\mathbb{P}_{E[10]^{(1)}}\left\{\mathrm{K}_{e}>0\right\}\right) \\
& +\lambda_{E[0]}^{(1)}\left(\mathbb{E}_{E[0]^{(1)}} \mathrm{K}_{e}-\mathbb{P}_{E[0]^{(1)}}\left\{\mathrm{K}_{e}>0\right\}\right) .
\end{aligned}
$$

The first term of the $\lambda_{E[2]}$ expression accounts for $\mathscr{Y}_{2-}$ genesis edges and the other three terms account for $\mathscr{Y}_{1}$ genesis edges.

Expressions above involving $\mathbb{P}_{X^{(1)}}\left\{\mathrm{K}_{x}>0\right\}$ or $\mathbb{P}_{X^{(1)}}\left\{\mathrm{K}_{x}=0\right\}$ can be evaluated in some models, notably those which have the 'Poisson Transect' property (see Remark 1). These expressions are also easy to evaluate if $\mathscr{Y}_{1}$ has no edges of types $E[10]$ and $E[0]$ (see the calculation for the $2 \times 1$-tiling).

Edge-types formed by nesting: With nested tessellations, the theory for edges of $\mathscr{Y}_{2}$-genesis has been dealt with in Lemma 3.

The number of hits by the edges of $\mathscr{Y} / 2$ on a typical edge $x$ in $\mathscr{Y}_{1}$ of type $X$ is denoted, as before, by $\mathrm{K}_{x}$. It now has expectation given by double the value in Eq. 31. The $\mathrm{K}_{x}$ hits partition the original type- $X$ edge into $\mathrm{K}_{x}+1$ edges, but an analysis of the types of edge $(E[2], E[11], E[10]$ or $E[0])$ is difficult, except in cases where $\mathscr{Y} / 2$ is a tessellation which has the Poisson Transect property that we mentioned in Remark 1 . The penultimate section gives such an example. 